\title{
Two-layer geostrophic vortex dynamics. Part 1. Upper-layer V-states and merger
}

\author{
By L. M. POLVANI ${ }^{1} \dagger$, N. J. ZABUSKY $Y^{2}$ AND G. R. FLIERL ${ }^{1}$ \\ ${ }^{1}$ Center for Meteorology and Physical Oceanography, Massachusetts Institute of Technology, \\ Cambridge, MA 02139, USA \\ ${ }^{2}$ Institute for Computational Mathematics and Applications, Department of Mathematics \\ and Statistics, University of Pittsburgh, Pittsburgh, PA 15260, USA
}

(Received 10 December 1987 and in revised form 11 January 1989)

We generalize the methods of two-dimensional contour dynamics to study a twolayer rotating fluid that obeys the quasi-geostrophic equations. We consider here only the case of a constant-potential-vorticity lower layer. We derive equilibrium solutions for monopolar (rotating) and dipolar (translating) geostrophic vortices in the upper layer, and compare them with the Euler case. We show that the equivalent barotropic (infinite lower layer) case is a singular limit of the two-layer system. We also investigate the effect of a finite lower layer on the merger of two regions of equalsign potential vorticity in the upper layer. We discuss our results in the light of the recent laboratory experiments of Griffiths \& Hopfinger (1986). The process of filamentation is found to be greatly suppressed for equivalent barotropic dynamics on scales larger than the radius of deformation. We show that the variation of the critical initial distance for merger as a function of the radius of deformation and the ratio of the layers at rest is closely related to the existence of vortex-pair equilibria and their geometrical properties.

\section{Introduction}

The role of coherent structures in the dynamics of two-dimensional vorticity fields has been the subject of many investigations in recent years. In particular, McWilliams (1984) has shown that, under certain conditions, coherent vortex structures emerge spontaneously from an initially random distribution of vorticity, and that, once formed, they play the dominant role in the subsequent evolution of the system.

One successful way of determining the shapes of coherent two-dimensional structures has been to approximate them by finite-area vortex regions of piecewiseconstant vorticity (vortex patches). Thus Deem \& Zabusky $(1978 a, b)$, Pierrehumbert (1980), Saffman \& Szeto (1980), Saffman \& Schatzman (1982) and Dritschel (1985) have numerically determined fully nonlinear steady-state (i.e. rotating or translating without change in shape) solutions of the inviscid Euler equations in two dimensions, while Norbury (1973) determined three-dimensional translating solutions with axisymmetry. These solutions have been designated 'V-states' (Vortex-states) by Deem \& Zabusky $(1978 a, b)$.

In two-dimensions, among the various types of fundamental interactions between vortices, it is the question of the merger of two regions of same-sign vorticity that has

$\dagger$ Present address: Department of Mathematics, Room 2-339, Massachusetts Institute of Technology, Cambridge, MA 02139, USA. 
received the greatest attention. The earliest studies (Zabusky, Hughes \& Roberts 1979; Deem \& Zabusky 1978 $a, b$; Overman \& Zabusky 1982a) used contourdynamical algorithms to investigate the evolution of two initially circular regions of constant vorticity. The same problem was considered with a perturbative approach, the moment model, by Melander, Zabusky \& Styczek (1986), and more recently revisited using pseudospectral methods (Melander, Zabusky \& McWilliams 1988). Several other types of interactions have also been examined, among which we mention asymmetric r.erger (Melander, Zabusky \& McWilliams 1987b), mergers arising from unstable perturbed $V$-states (Overman \& Zabusky 1982a) and scattering of translating V-states (Overman \& Zabusky 1982b). The question of axisymmetrization has also been investigated. Melander, McWilliams \& Zabusky (1987 $a)$ have shown how an initially non-axisymmetric vorticity distribution will axisymmetrize through the process of filamentation. Their pseudospectral calculations have been supported, for short times, by the contour dynamical computations of Dritschel (1988).

The applicability of all of the above studies to geophysical flows is, however, limited by the fact that they treat exclusively two-dimensional systems. It is then a question of major concern to investigate if and to what extent the ideas that have emerged from the investigation of purely two-dimensional problems generalize to three-dimensional situations. With this in mind we have taken what we believe to be the simplest step away from two-dimensionality by turning our attention to the easiest possible two-layer system.

The so called Phillips' model (Phillips 1954) describes the evolution of a two-layer rotating fluid in the limit of small Rossby number (i.e. in geostrophic balance), and is therefore of particular relevance for geophysical flows. It is the simplest system that incorporates the properties of both rotation and stratification, and thereby can support baroclinic instability, which is an essentially three-dimensional phenomenon. Gryanic (1983), Hogg \& Stommel (1985) and Young (1985) have studied the pointvortex solutions of the two-layer model and have investigated their interactions. Griffiths \& Hopfinger (1986) have recently used the same model to interpret their laboratory experiments with a rotating stratified fluid. In particular, they have conducted experiments on the coalescence of two circular vortices in the upper layer (Griffiths \& Hopfinger 1987).

Generalizing the contour-dynamics method to the Phillips' model, we have been able to address some of the fundamental questions that have already found an answer in the purely two-dimensional case. In particular we have found a variety of two-layer rotating and translating $V$-states. We have also examined in some detail the interaction of two like-signed vortices. Since the two vortices can be either in the same layer or in different layers, two distinct processes need to be identified. In the former case we designate the coalescence of two vortices with the name 'merger', by analogy with the two-dimensional case.

When the vortices coalesce from different layers we refer to their interaction with the term 'alignment' since, in recent high-resolution numerical simulations of stratified geostrophic turbulence, McWilliams (1989), has shown that vorticity in different layers tends to align in the vertical direction, yielding columnar vortex structures. In the language of turbulence theories, it should be clear that, in the same way as the merger process mediates the well-known reverse energy cascade of twodimensional turbulence, the alignment process is the fundamental mechanism for the cascade from baroclinic to barotropic modes (see, for instance, Salmon 1982). The 
alignment process has been studied in detail (Polvani 1988), and will be the subject of a second paper.

In this first paper, we consider only the simplest possible departure from twodimensionality, and restrict our attention to vorticity fields in which the potential vorticity in the lower layer is uniform. We present here a variety of upper-layer $V$ states and our results for the investigation of the merger problem. We start with a general review of how to extend the method of contour dynamics to the two-layer model.

\section{Contour dynamics for the two-layer model}

The quasi-geostrophic two-layer model (Phillips' model) has been used extensively in the oceanographic and meteorological literature, and it will suffice here to recall its principal characteristics (for a full derivation see Pedlosky 1979). As shown in figure 1, the system is composed of two horizontal layers of density $\rho$ and $\rho+\Delta \rho$, where $\Delta \rho \ll \rho$. The thickness $H_{1}$ (and $H_{2}$ ) of the upper (and lower) layer is considered to be much smaller than the horizontal scale $L$ of the system, so that the fluid is always in hydrostatic equilibrium. The system rotates with an angular velocity $\omega$ large compared with the relative vorticity $U / L$ ( $U$ being the velocity scale), i.e. the Rossby number $R o=U /(2 \omega L)$ is small compared to 1 .

Under these assumptions, the motion in each layer is two-dimensional (cf. the Taylor-Proudman theorem) and can be described by a stream function $\psi_{i}(i=1,2)$. The dynamics of the system is dictated by the statement of conservation of the geostrophic potential vorticity $\Pi_{i}$ in each layer. In non-dimensional form, the two-layer model equations take the simple form

$$
\left[\partial_{t}+J\left(\psi_{i}, \cdot\right)\right] \Pi_{i}=0 \text { for } i=1,2,
$$

where $\Pi_{i}$ is defined by

$$
\Pi_{1}=\nabla^{2} \psi_{1}+\gamma^{2}\left(\psi_{2}-\psi_{1}\right), \quad \Pi_{2}=\nabla^{2} \psi_{2}+\delta \gamma^{2}\left(\psi_{1}-\psi_{2}\right)
$$

with

$$
J(A, B)=\partial_{x} A \partial_{y} B-\partial_{y} A \partial_{x} B \text {. }
$$

Note that only two non-dimensional parameters appear in this formulation: $\delta=$ $H_{1} / H_{2}$ is the ratio of the upper to lower layer thickness (when the fluid is at rest) and $\gamma=L / L_{R}$, where

$$
L_{R}=(2 \omega)^{-1}\left(\frac{g \Delta \rho H_{1}}{\rho}\right)^{\frac{1}{2}}
$$

is the radius of deformation for the upper layer. The parameter $\gamma$ can be intuitively understood as the rigidity of the interface. In particular when $\gamma=0$ (which corresponds to a perfectly rigid interface) the two layers become uncoupled, and behave as two independent two-dimensional systems obeying the Euler equations.

The principal limitation of this model rests in the fact that the layer thicknesses are only allowed to change by an $O(R o)$ amount with respect to their values when the fluid is at rest. Thus a phenomenon such as the formation of fronts is totally absent in this approximation. This model represents the stratification by two coupled layers in which the advection is two-dimensional, and it retains the linear relationship between the stream function and the geostrophic potential vorticity, as is apparent in (2). A direct analogy can therefore be drawn with the two-dimensional Euler 


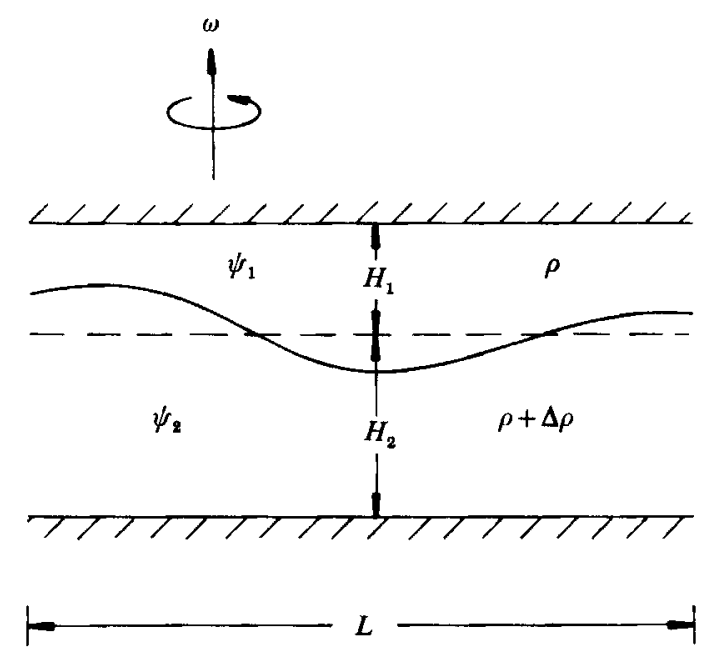

Figure 1. The quasi-geostrophic two-layer model.

equations, were the vorticity $\nabla^{2} \psi$ plays the role of the geostrophic potential vorticity $I I$.

Consider then a field made up of $N_{j}$ regions $D_{j}^{(n)}(j=1,2$ refers to the upper and lower layer respectively, and $n=1, N_{j}$ ) of constant geostrophic potential vorticity $\Pi_{j}^{(n)}$. It is easy to see that the stream function in layer $i$ can be obtained from the Biot-Savart-type formula:

$$
\psi_{i}(x, y)=\frac{1}{2 \pi} \sum_{j=1}^{2} \sum_{n=1}^{N_{j}} \Pi_{j}^{(n)} \iint_{D_{j}^{(n)}} G_{i j}(r) \mathrm{d} \xi \mathrm{d} \eta
$$

where

$$
r=\left[(x-\xi)^{2}+(y-\eta)^{2}\right]^{\frac{1}{2}}
$$

and $G_{i j}$ is the Green function for the effect in layer $i$ of a point vortex in layer $j$. The four different Green functions needed can be derived in a straightforward way from (2) and are found to be

$$
\begin{array}{ll}
G_{11}=\frac{\delta}{1+\delta} \log r-\frac{1}{1+\delta} K_{0}(\Gamma r), \quad G_{21}=\frac{\delta}{1+\delta} \log r+\frac{\delta}{1+\delta} K_{0}(\Gamma r), & (4 a, b) \\
G_{12}=\frac{1}{1+d} \log r+\frac{1}{1+\delta} K_{0}(\Gamma r), & G_{22}=\frac{1}{1+d} \log r-\frac{\delta}{1+\delta} K_{0}(\Gamma r),
\end{array}
$$

where

$$
\Gamma=\gamma(1+\delta)^{\frac{1}{2}}
$$

and $K_{0}$ is the modified Bessel function of order zero. Differentiating (3) and applying Green's theorem, one obtains expressions for the velocities in layer $i$ as contour integrals over the boundaries $\partial D_{j}^{(n)}$ of the regions of constant geostrophic potential vorticity :

$$
\left[u_{i}, v_{i}\right]=\left[-\partial_{y} \psi_{i}, \partial_{x} \psi_{i}\right]=-\frac{1}{2 \pi} \sum_{j=1}^{2} \sum_{n=1}^{N_{j}}\left\{\Pi_{j}^{(n)}\right\} \int_{\partial D_{j}^{(n)}} G_{i j}(r)[\mathrm{d} \xi, \mathrm{d} \eta] .
$$

where $\left\{\Pi_{i}^{(n)}\right\}$ is the jump in geostrophic potential vorticity (outside-inside) at the boundary $\partial D_{j}^{(n)}$. Having derived the basic formulas for the full two-layer model, we now restrict our attention to the case for which $\Pi_{2}=0$ everywhere.

This situation constitutes the simplest departure from a purely two-dimensional situation. Note that, although it has zero geostrophic potential vorticity (i.e. 
constant total potential vorticity equal to $2 \omega / H_{2}$ ), the lower layer is not at rest (i.e. $\psi_{2} \neq 0$ ) unless $\delta=0$, and the objective of this paper is to understand the effect of the lower-layer motion on the evolution of the vorticity in the upper layer. Since the geostrophic potential vorticity resides uniquely in this layer, and since there is no need to calculate the fields in the lower layer to follow the evolution of the vorticity in the upper layer, as can be seen from (5), we can imagine dealing with a purely twodimensional system (the upper layer) in which vortices interact with a modified Green function $G_{11}$ given by $(4 a)$, instead of the simple logarithm.

Before proceeding to present our results for the upper-layer $V$-states and merger, it is of great interest to examine carefully the limiting forms of $G_{11}$ as $\gamma$ is varied from zero to infinity. When $\delta \neq 0$, i.e. in the presence of a finite lower layer, it is easily seen that

$$
\lim _{\gamma \rightarrow 0} G_{11}=\log (r), \quad \lim _{\gamma \rightarrow \infty} G_{11}=\frac{\delta}{1+\delta} \log (r) \text { for } \delta \neq 0
$$

(ignoring terms independent of $r$ which do not affect the velocities). Thus, as long as the lower layer is finite, i.e. $\delta \neq 0$, we can expect the dynamics to be identical to twodimensional Euler dynamics in both limits, with the exception that, for every large $\gamma$, time is rescaled by a factor of $\delta(1+\delta)^{-1}$.

Of special interest is the case $\delta=0$, for which the lower layer is infinitely deep. This situation is commonly referred to in the literature as the 'equivalent barotropic' model (or the 'reduced gravity' model, or again the 'quasi-geostrophic shallow-water model'). It has been widely used in oceanographic and meteorological contexts to study the effect of density stratification without explicitly introducing a second layer. Since

$$
G_{\text {e.b. }}=G_{11}(\delta=0)=-K_{0}(\gamma r)
$$

the large- $\gamma$ limit in the equivalent barotropic case is given by

$$
\lim _{\gamma \rightarrow \infty} G_{\text {e.b. }}=\left[\frac{\pi}{2 \gamma r}\right]^{\frac{1}{2}} \mathrm{e}^{-\gamma r}
$$

Thus, at large $\gamma$ the equivalent barotropic model exhibits singular behaviour, in as much as (6) is qualitatively very different from the logarithmic type of interaction that would occur if the lower layer were finite. This exponential decay underlies one of the major findings of this study (preliminarily reported in Polvani, Zabusky \& Flierl 1988), namely that the dynamics of vortices in the equivalent barotropic model at large $\gamma$ is in many respects very different from those of a two-layer system with a deep lower layer of zero geostrophic potential vorticity.

To illustrate this point we have plotted in figure $2(a)$ the function $G_{11}(r)$ vs. $r$ for $\delta=0,0.2$ and 1 and, in figure $2(b)$, the azimuthal velocity due to a point vortex in the upper layer at $\gamma=5$. Note how $G_{11}$ diverges logarithmically when $\delta \neq 0$, but is cut off exponentially for the equivalent barotropic case.

In practice, as will be shown in what follows, $\gamma$ does not have to be very large before the different behaviour appears. We have found that for motions on scales only a few times the radius of deformation, the results obtained from the equivalent barotropic model are remarkably different from those of a two-layer model with a deep but finite lower layer.

An alternative way to understand how this arises is to consider the competition of the two terms in $(4 a)$ when $\delta \ll 1$ and $\gamma \gg 1$ at the same time. It is easy to see that the modified Bessel term will dominate when $\delta<O\left(\gamma^{-\frac{1}{2}} \mathrm{e}^{-\gamma}\right)$. In other words, the equivalent barotropic model reproduces the dynamies of a two-layer system with a 
(a)

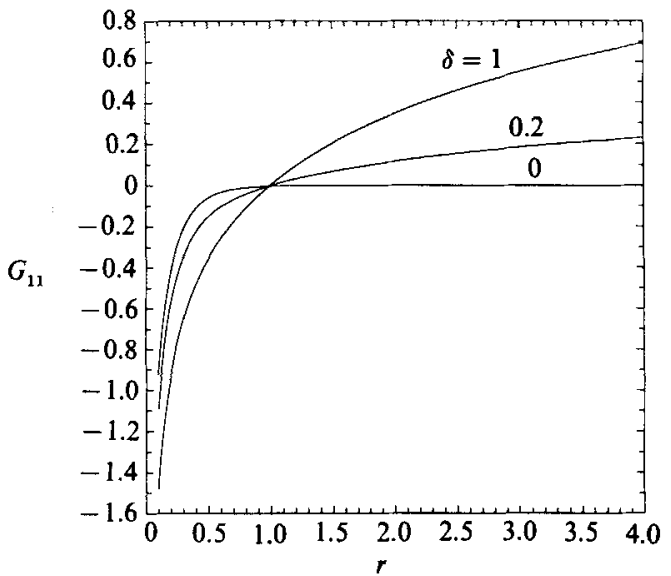

(b)

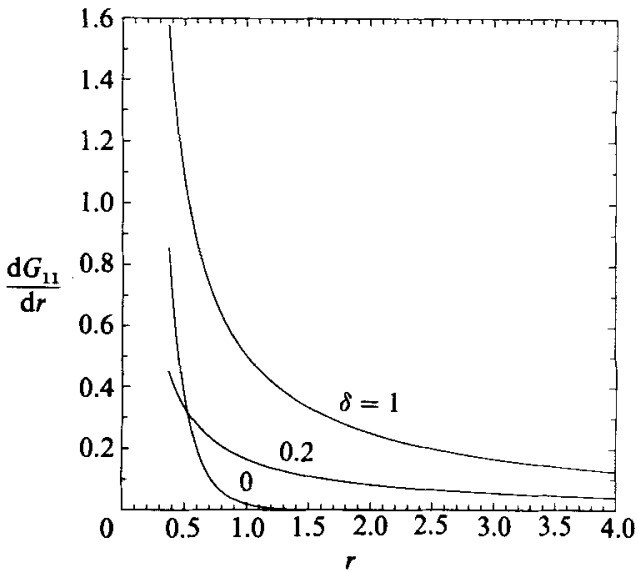

Figure 2. Plots of $(a)$ the Green function $G_{11}(r)$ and $(b)$ its derivative $\mathrm{d} G_{11} / \mathrm{d} r$, for $\gamma=5$.

lower layer $O\left(\gamma^{\frac{1}{2}} \mathrm{e}^{\gamma}\right)$ deeper than the upper one. Because this number increases exponentially with $\gamma$, it is clear that the equivalent barotropic approximation at large $\gamma$ should be used with care for most oceanic and atmospheric systems, for which the relevant value of $\delta$ is $O(1)$.

\section{Rotating V-states}

We now turn our attention to the $m=2$ rotating $V$-states. As shown in figure 3 , the upper layer consist of a patch of uniform geostrophic potential vorticity (without loss of generality we choose it to have value 1) of aspect ratio $\lambda=R_{a} / R_{b}$ (again without loss of generality we choose $R_{b}=1$, which is equivalent to setting $\gamma=$ $R_{b} / L_{R}$ ) rotating with constant angular velocity $\Omega$ without change in shape in a background of zero geostrophic potential vorticity in the upper layer. The problem consists in determining the shape of the boundary, and the angular velocity $\Omega$. To do this we use the second-order algorithm of Wu, Overman \& Zabusky (1984), with the modification that the logarithmic Green function of the Euler equations is replaced by $G_{11}$. We present only a very brief summary of that method here, and for a full exposition we refer the reader to $\mathrm{Wu}$ et al. (1984).

By symmetry, only one quarter of the contour need be considered. It is discretized by $N$ nodes placed at equal angular positions between 0 and $\frac{1}{2} \pi$ (the results presented were obtained with $N=75$ ). The unknowns are the radial distances $R_{i}(i=1, N)$ from the origin to the nodes and the angular velocity $\Omega$. One starts from an initial guess for the $R_{i}$ and $\Omega$ and calculates the velocities at each node using a discretized version of (5). The new values of the $R_{i}$ and $\Omega$ are then obtained by enforcing the condition that the velocity be tangent to the boundary in the frame of reference rotating with the $V$-state. From these new values, new velocities are calculated, and the process is repeated until some convergence criterion is satisfied. In practice, as was the case for Wu et al. (1984), a relaxation scheme is needed to ensure a convergence of the algorithm, i.e. at each step the new $R_{i}$ are 'mixed' with a certain proportion of the previous ones.

Since for each value of $\gamma, \delta$ and $\lambda$ a $V$-state can be determined, the parameter space for this problem is three-dimensional, and an extraordinary amount of calculations would be necessary for its full exploration. We have decided to select the minimum 


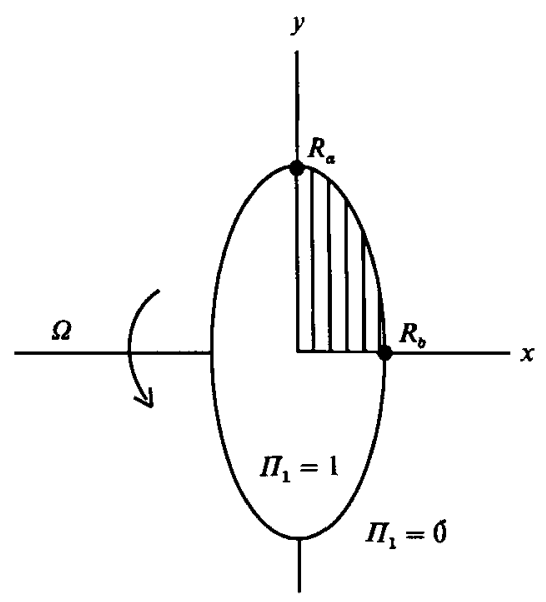

FIGURE 3. Schematic drawing of the geostrophic potential vorticity distribution in the upper layer for a monopolar rotating $(m=2) \mathrm{V}$-state.

number of cases that would allow us to grasp the qualitative behaviour of the solutions as the parameters are varied. We have selected three values of the aspect ratio $\lambda(1.5,2.5$ and 3.5$)$ and, for each, three values of $\delta$, the ratio of the layers' depths at rest; $\delta=0$ is an obvious choice, since it represents the equivalent barotropic model; the other obvious choice is $\delta=1$ (layers of equal depth); finally $\delta=0.2$ was chosen as an intermediate value, since it roughly corresponds to the ratio of the depth of the oceanic thermocline to the full depth of the ocean (in the simplest nonconstant-density approximation the ocean is often modelled by a two-layer system, with the interface between the two layers representing the thermocline).

For each value of $\lambda$ and $\delta$ we have determined the $V$-state solutions for 21 values of $\gamma$ that span in approximately equal intervals (on a logarithmic scale) the four orders of magnitude $0.01 \leqslant \gamma \leqslant 100$. The reason for extending our investigation to such high and low values of $\gamma$ is that we want to ensure that we recover the asymptotic results known for the limits $\gamma \ll 1$ and $\gamma \gg 1$. Indeed, since in those limits, as was shown earlier, the Green function reduces to a pure logarithm (except for the case $\delta=0$ ), we expect the $V$-states to reduce to ellipses, following Kirchhoff's arguments.

The problem can then be restated in the following simple form: if $\gamma$ is varied between zero and infinity, how do the $m=2$ rotating $V$-states differ from Kirchhoff ellipses? How does this difference depend on $\delta$ and $\lambda$ ? The answer is presented in figures 4,5 and 6 for $\lambda=1.5,2.5$ and 3.5 respectively.

Consider first figure 4 in which the aspect ratio $\lambda$ is held fixed and equal to 1.5. In figures $4(a), 4(b)$ and $4(c)$ we have plotted one quarter of the equilibrium shapes for $\delta=0,0.2$ and 1 respectively; for clarity only the $V$-states for $\gamma=0.01,1$ and 10 are shown (the solid lines represent the Kirchhoff ellipses). The first remarkable result is that, for small aspect ratio, over the whole range of $\gamma$ the shapes are extremely close to Kirchhoff ellipses.

To quantify this we have calculated the quantity $\delta A$ which represents the fractional change in area between the $V$-states and the Kirchhoff ellipse, or more precisely

$$
\delta A=\frac{A_{\mathrm{K}}-A}{A_{\mathrm{K}}}
$$



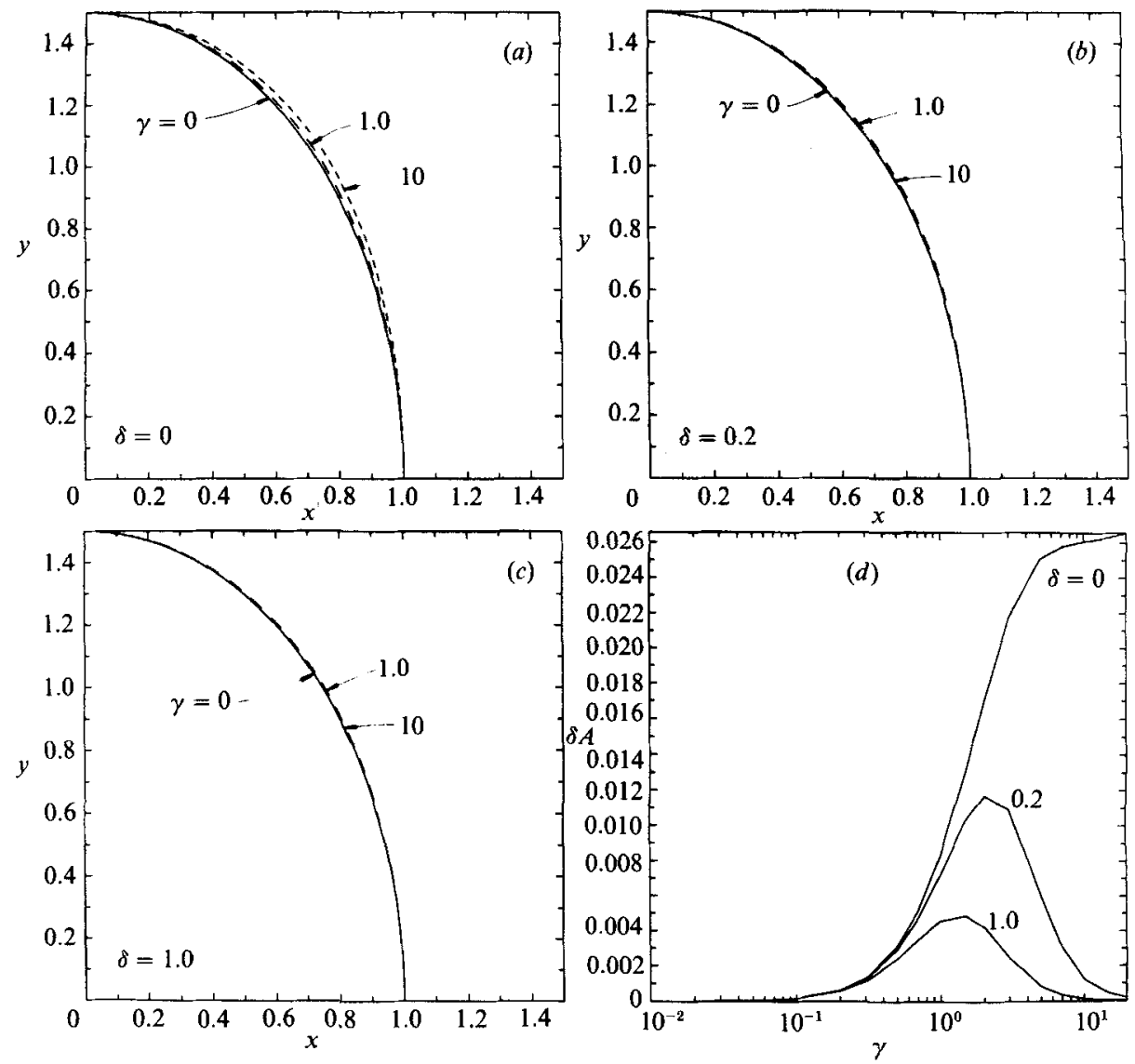

Figure 4. Rotating one-layer $V$-states of aspect ratio $\lambda=1.5$, for $\delta=0,0.2$ and 1,0. Only the hatched sector in figure 3 is shown.

where $A_{\mathrm{K}}$ and $A$ are the area of the Kirchhoff ellipse and of the $\mathrm{V}$-state respectively. In figure $4(d)$ we plot $\delta A$ as function of $\gamma$ for the three values of $\delta$. It is immediately apparent that the case $\delta=0$ is qualitatively different from the cases for which $\delta$ is finite. This reflects the singular behaviour of the equivalent barotropic model at large $\gamma$ that we predicted from the analysis of the Green function. It is, however, interesting to note that, even for a lower layer considerably deeper than the upper one (5 to 1 ), the fractional change in area barely exceeds $1 \%$ over the whole range of $g$ for this relatively small value of the aspect ratio $\lambda$.

For the slightly higher value of $\lambda=2.5$, the results are shown in figure 5. To keep the figures readable, for each $d$ we show only a few $V$-states chosen to represent the variations in shape over the whole $\gamma$-range; in particular the $V$-states for $\gamma=0.01$ and 10 are always shown. Again the considerable insensitivity of the Kirchhoff ellipse to the presence of a lower layer is manifested in the fact that the maximum fractional change in area for $\delta=0.2$ is of only a few percent even for this reasonably high value of the aspect ratio ( 5 to 2 ). The anomalous behaviour of the $\delta=0$ case is reflected in the fact that, as $\gamma$ is increased, the shape never reverts back to the Kirchhoff ellipse. We have found that, at large $\gamma$, our algorithm requires many more iterations to converge in the equivalent barotropic case than when $\delta \neq 0$, and we attribute this to the fact that the equilibrium shapes have both positive and negative curvatures. 

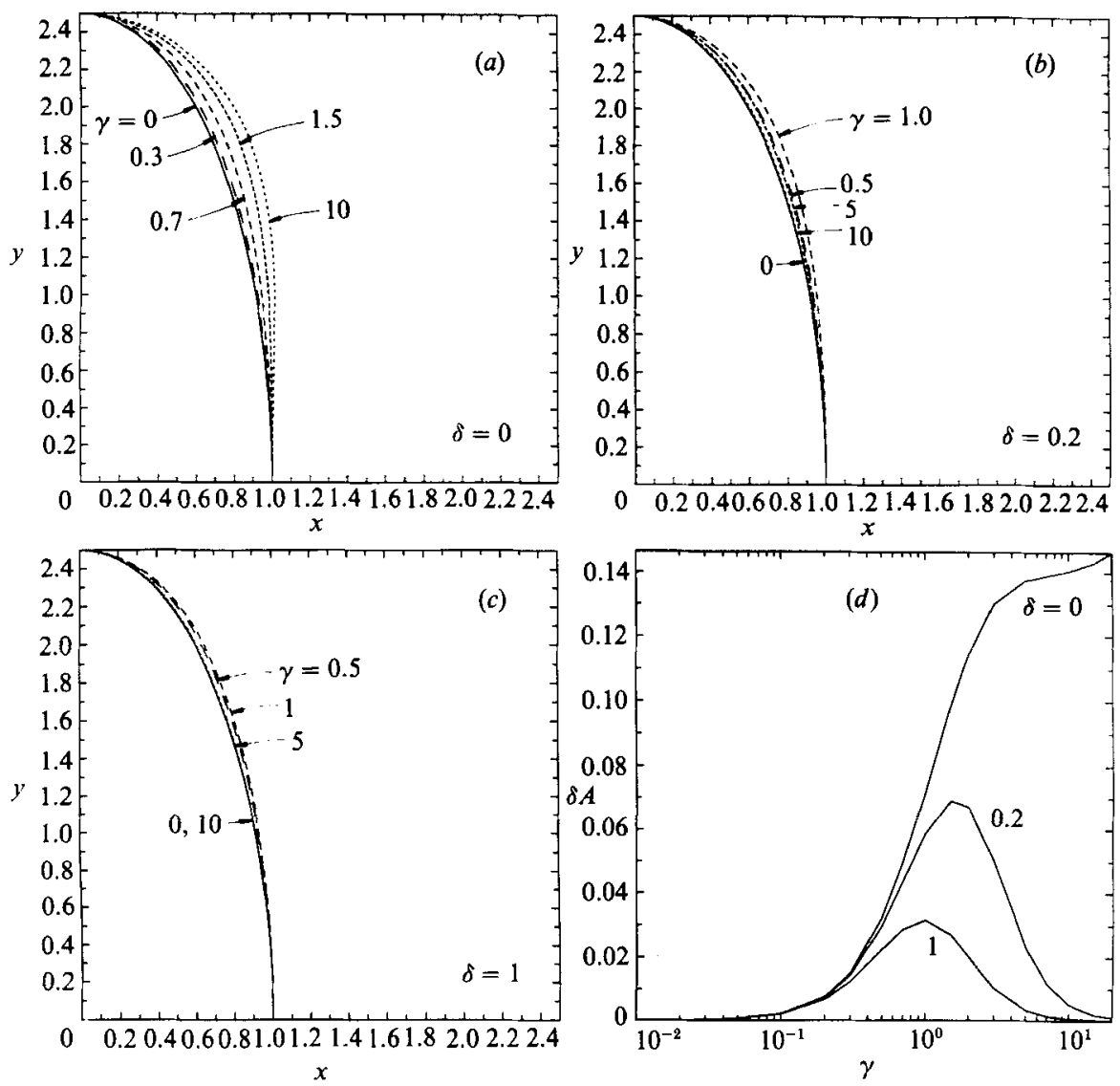

FIGURE 5. Rotating one-layer V-states of aspect ratio $\lambda=2.5$, for $\delta=0,0.2$ and 1.0 .

This can be seen very clearly in figure $6(a)$ in which the aspect ratio $\lambda=3.5$. For $\delta=0$, as $\gamma$ is increased from zero, the $\mathrm{V}$-states bulge away from the Kirchhoff ellipse and eventually become 'peanut' shaped. This singular behaviour is, however, restricted to the equivalent barotropic case and for $\delta \neq 0$ (figure $6 b, c$ ) the conclusion is the same as the one reached for the previous two values of $\lambda$, namely that over the entire $\gamma$-range the $V$-states are remarkably close to Kirchhoff ellipses, even for quite large aspect ratios.

In figures $7(a), 7(b)$ and $7(c)$ we have plotted the values of the angular velocity $\Omega$ of the $V$-states as a function of $\gamma$ for $\lambda=1.5,2.5$ and 3.5 respectively. For $\delta \neq 0$, both the small- and large- $\gamma$-limits are finite and are given by

$$
\lim _{\gamma \rightarrow 0} \Omega=\Omega_{\mathrm{K}}, \quad \lim _{\gamma \rightarrow \infty} \Omega=\frac{\delta}{1+\delta} \Omega_{\mathrm{K}} \quad \text { for } \quad \delta \neq 0,
$$

where

$$
\Omega_{\mathrm{K}}=\lambda /(1+\lambda)^{2}
$$

is the angular velocity for a Kirchhoff ellipse of aspect ratio $\lambda$.

In the equivalent barotropic case $\delta=0$, however, the angular velocity goes to zero as $\gamma$ becomes very large. This is easily understood by recalling that, in that limit, the Green function is a rapidly decaying exponential.

We have fitted the $\log -\log$ curves of $\Omega$ vs. $\gamma$ at large $\gamma$ with straight lines, and the 

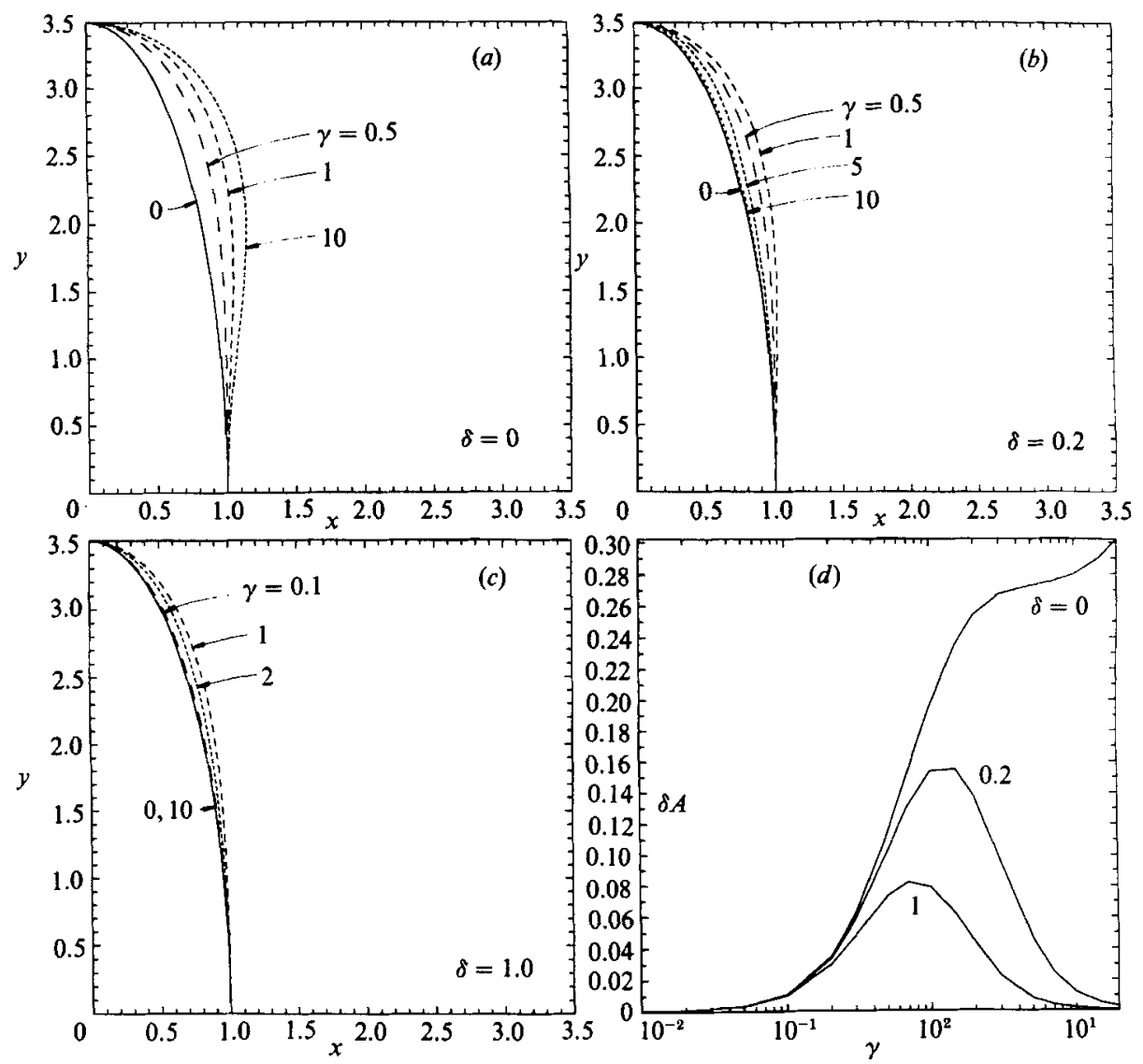

Figure 6. Rotating one-layer $V$-states of aspect ratio $\lambda=3.5$, for $\delta=0,0.2$ and 1.0 .

slopes were found to be $2.94,2.93$ and 2.89 for $\lambda=1.5,2.5$ and 3.5 respectively. This strongly suggests that in developing an asympotic theory of the equivalent barotropic equations at large $\gamma$ a slow timescale ought to be chosen to be proportional to $\gamma^{3}$. Pratt \& Stern (1986), in considering the long-wave approximation (i.e. $\gamma \gg 1$ ) of a perturbation on a vorticity front in an equivalent barotropic model, found that a dominant balance could be reached only when time was scaled like $\gamma^{3}$. However, their derivation seems to us less transparent than it might be, in as much as it is rather hard to see why such a scaling arises naturally. A much simpler derivation of the equivalent barotropic long-wave equation is presented here in the Appendix.

It is simple to derive the $\gamma^{3}$ scaling law for time at large $\gamma$ by considering how the kinematic boundary condition is satisfied at the edge of the $V$-states. Let $R(\theta)$ be the radial distance of the boundary from the origin. Then the dimensional streamfunction $\Psi$ must satisfy

$$
\frac{\partial}{\partial t} R=\frac{1}{R} \frac{\partial}{\partial \theta} \Psi
$$

for all angles $\theta$ on the boundary. From the asymptotic analysis one learns that, at leading order in $1 / \gamma, \Psi$ is independent of $\theta$, and that it is the $O(1 / \gamma)$ correction to $\Psi$ which allows the boundary condition to be satisfied in time. In order to determine the scale for $t$ we define

$$
R=L R^{*}, \quad \Psi=q L_{R}^{2} \frac{1}{\gamma} \Psi^{*}, \quad t=\gamma^{n} q^{-1} t^{*}
$$


(a)

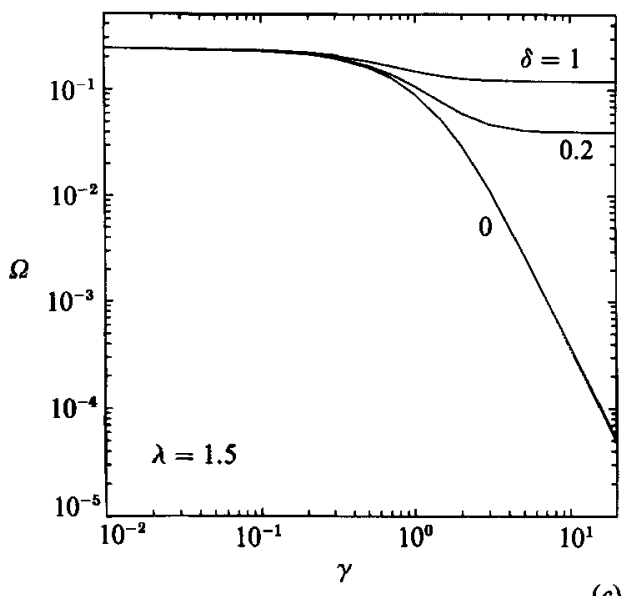

(b)

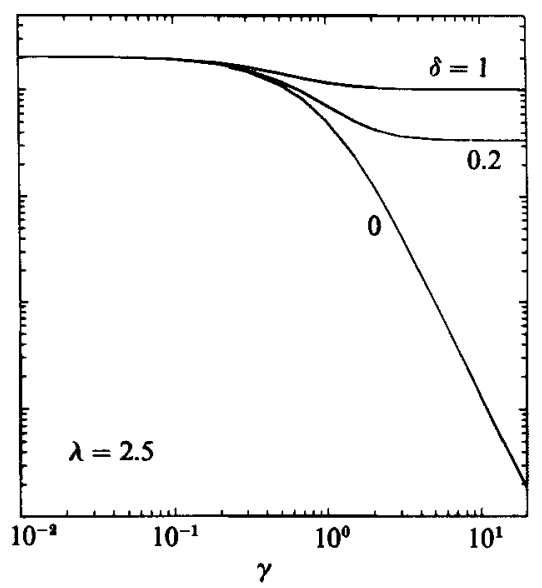

(c)

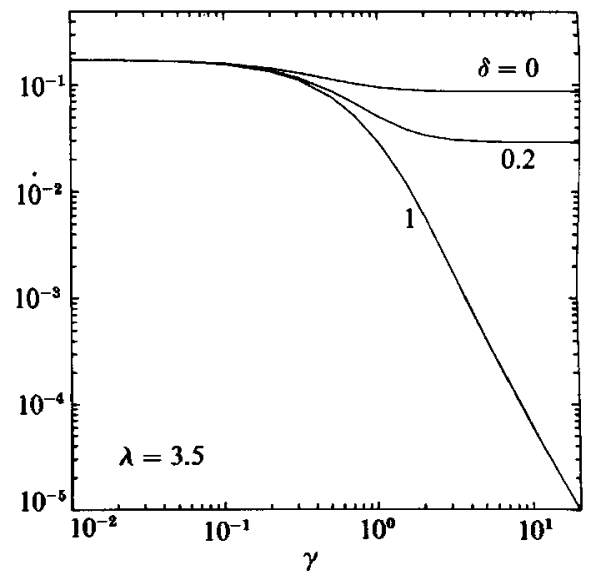

Figure 7. The angular velocity $\Omega$ of rotating $V$-states as a function of the radius of deformation $\gamma$.

where the starred quantities are non-dimensional and $O(1)$, and $q$ is the vorticity scale. $\Psi$ is scaled one order smaller than the leading-order geostrophic stream function. Substituting these scalings in the above boundary condition, and requiring that the left- and right-hand sides be of same order yields the value $n=3$.

\section{Translating V-states}

The conclusions that were drawn from the analysis of the $m=2$ rotating $V$-states are confirmed by the properties of the translating ones, for which a schematic drawing is given in figure 8. Two regions of equal and opposite geostrophic potential vorticity propagate in a straight line along the $y$-axis with constant velocity $V$ without change in shape. Each state is characterized by an inner and an outer radius, $x_{A}$ and $x_{B}$ respectively. Without loss of generality we choose $x_{B}=1$, and define the parameter $\mu=x_{A} / x_{B}$. As in the rotating case, by symmetry only one quarter of the state need be considered and is discretized with $N$ nodes. The algorithm is identical to the one described above, with the exception of the boundary condition which has to be modified appropriately (see $\mathrm{Wu}$ et al. 1984). 


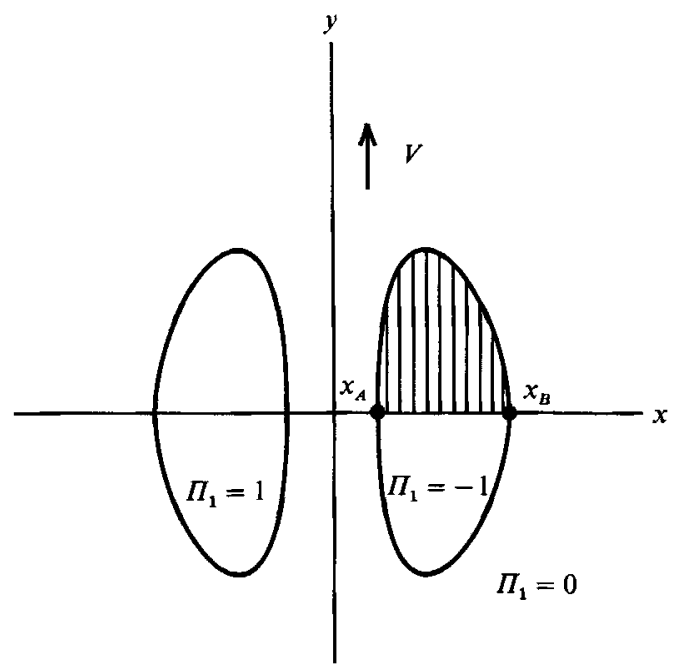

Figure 8. Schematic drawing of the geostrophic potential vorticity distribution in the upper layer for a translating $\mathrm{V}$-state.
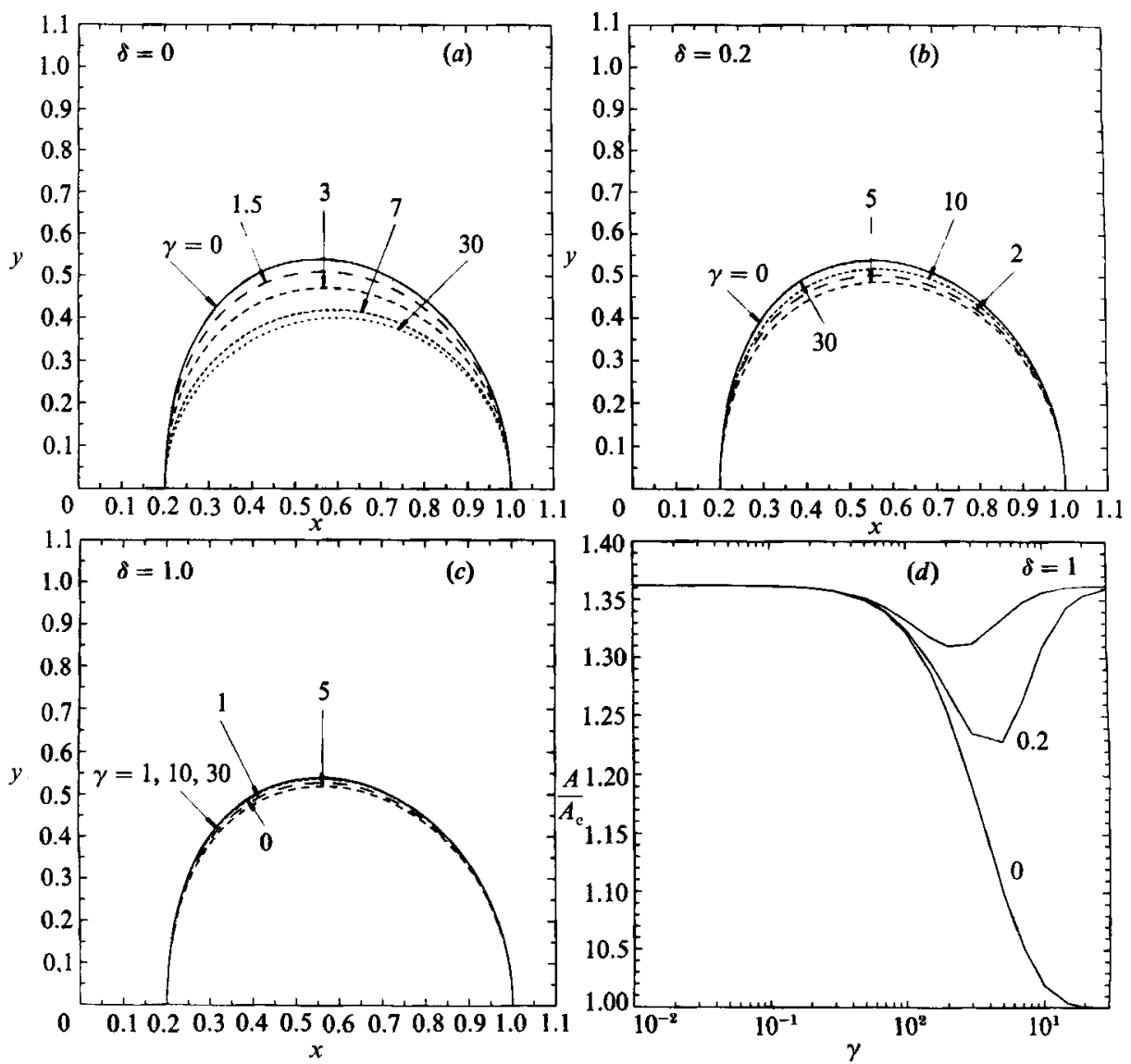

Figure 9. Translating one-layer $V$-states for $\mu=0.2$, and $\delta=0,0.2$ and 1.0. Only the hatched sector in figure 8 is shown. 

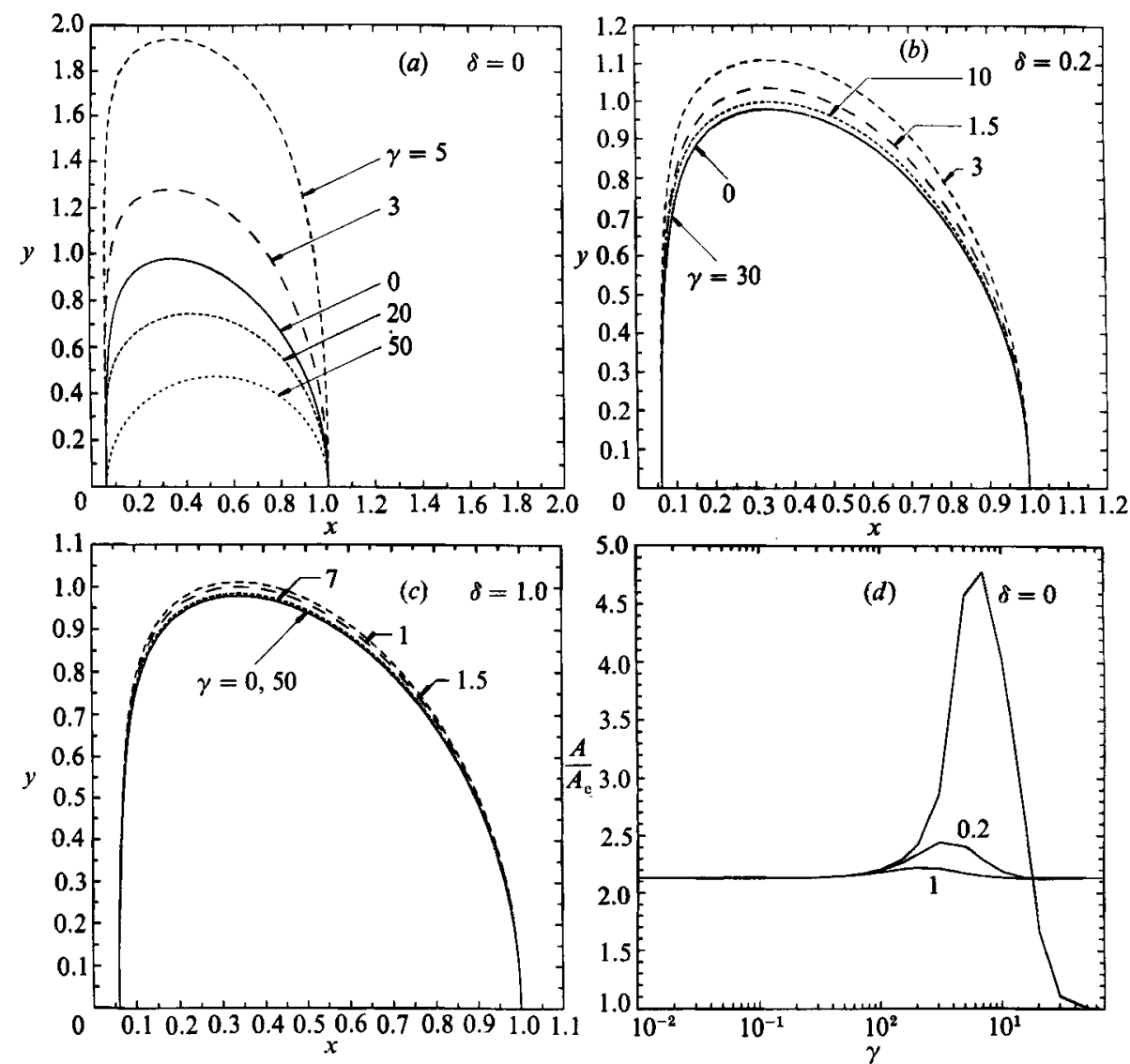

Figure 10. Translating one-layer V-states for $\mu=0.06$, and $\delta=0,0.2$ and 1.0 .

For the dipolar states, however, the limiting cases are not known analytically, as was the case in the previous section. In the $\gamma=0$ limit the translating Euler $V$-states of Deem \& Zabusky $(1978 a, b)$ are recovered; these are also the limiting states for $\gamma \gg 1$ (with the velocity scaled by $\delta /[1+\delta]$ ) provided $\delta \neq 0$. For the equivalent barotropic model in the large- $\gamma$ limit, the dipole tends to a pair of circles with $V=0$. We have used the same values of $\gamma$ and $\delta$ as for the rotating states to determine how much the solutions differ from the Euler solutions as $\gamma$ is varied from zero to infinity.

Figures $9(a), 9(b)$ and $9(c)$ show the results obtained with $N=75$ for $\mu=0.2$ and $\delta=0,0.2$ and 1 respectively (the solid curves are for $\gamma=0$ ). The equivalent barotropic solution relaxes monotonically from the Euler dipole to a circle. When $\delta \neq 0$ the deviations from the Euler solutions are not very large, as was the case for the translating $V$-states. Note that, for this value of $\mu$, the solutions always have a smaller aspect ratio than the Euler solutions. In figure $9(d)$ we have plotted the quantity $A / A_{\mathrm{c}}$, where $A$ is the area of one half of the $V$-state and $A_{\mathrm{c}}$ is the area of a circle of radius $\frac{1}{2}\left(1-x_{A}\right)$.

When the ratio $\mu$ of the inner to outer radii becomes smaller than a critical value (about 0.09 ) the behaviour becomes qualitatively different. As $\gamma$ is increased from zero the $V$-states become more elongated than the Euler ones. The case $\mu=0.06$ is shown in figure 10. Note that for the equivalent barotropic case the maximum 
(a)

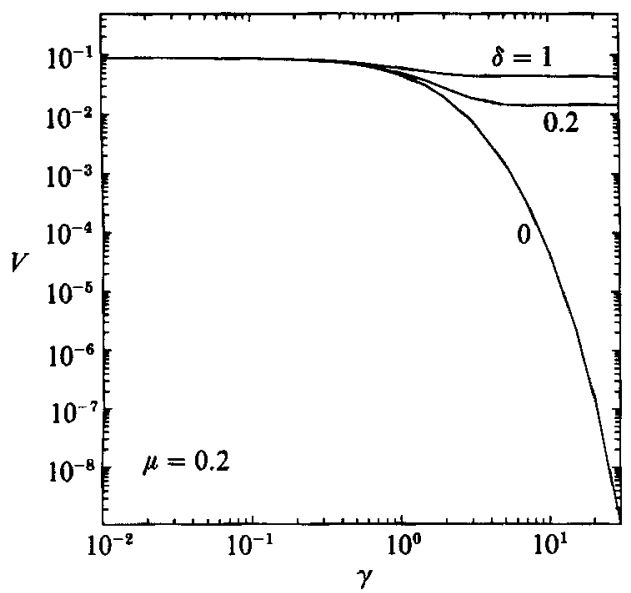

(b)

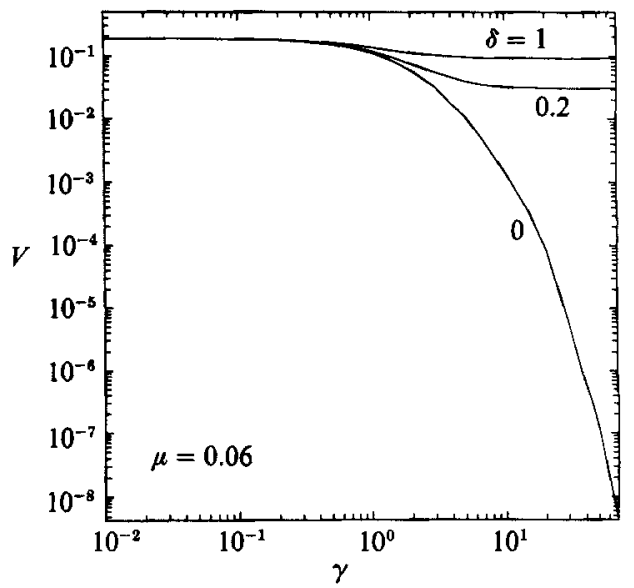

FigUre 11. The velocity $V$ of translating $V$-states as a function of the radius of deformation $\gamma$.

deformation which occurs at approximately $\gamma=7$ is very large (the area essentially doubles, cf. figure $9 d$ ). Finally, in figures $11(a)$ and $11(b)$ the velocity is plotted versus $\gamma$ for $\mu=0.2$ and 0.06 respectively. Again the behaviour is qualitatively the same as was observed for the rotating states.

\section{Merger in the two-layer model}

As was mentioned in the introduction the question of merger of two regions of equal-sign vorticity has been extensively investigated for the two-dimensional Euler equations (Melander et al. 1988). The initial vorticity distribution is taken to be two circular regions of equal and constant vorticity whose centres are separated by a distance $d_{\mathrm{c}}$. The time evolution of such an initial configuration falls into either of two qualitatively distinct behaviours: if the initial distance exceeds the critical nondimensional value of approximately 3.3 (the radius of the circles being the lengthscale), the two regions rotate about a common centre and pulsate as they revolve around each other. When, however, the initial distance is smaller than that critical value the two vortices approach each other and 'merge' forming a double spiral, and the final state is composed of a single vortex resulting from the coalescence of two originally distinct regions of vorticity.

Recently, Griffiths \& Hopfinger $(1986,1987)$ have conducted laboratory experiments with one- and two-layer rotating fluids to study the interactions of finite-area geostrophic vortices. For the purely barotropic case they were able to verify experimentally the value of 3.3 for the critical distance of vortex merger. Of interest to us here are the experiments on merger of two vortices in the upper layer of a two-layer rotating fluid.

For simplicity, Griffiths \& Hopfinger chose to consider a configuration in which the layers had equal depth. The values of the density stratification and of the rotation of the tank were chosen in such a way that the system was expected to obey the quasi-geostrophic two-layer equations (1). The authors in fact tested this assumption by analysing carefully the velocity field of a single circular vortex and comparing it with the theoretical prediction (for a circular geostrophic potential vorticity 


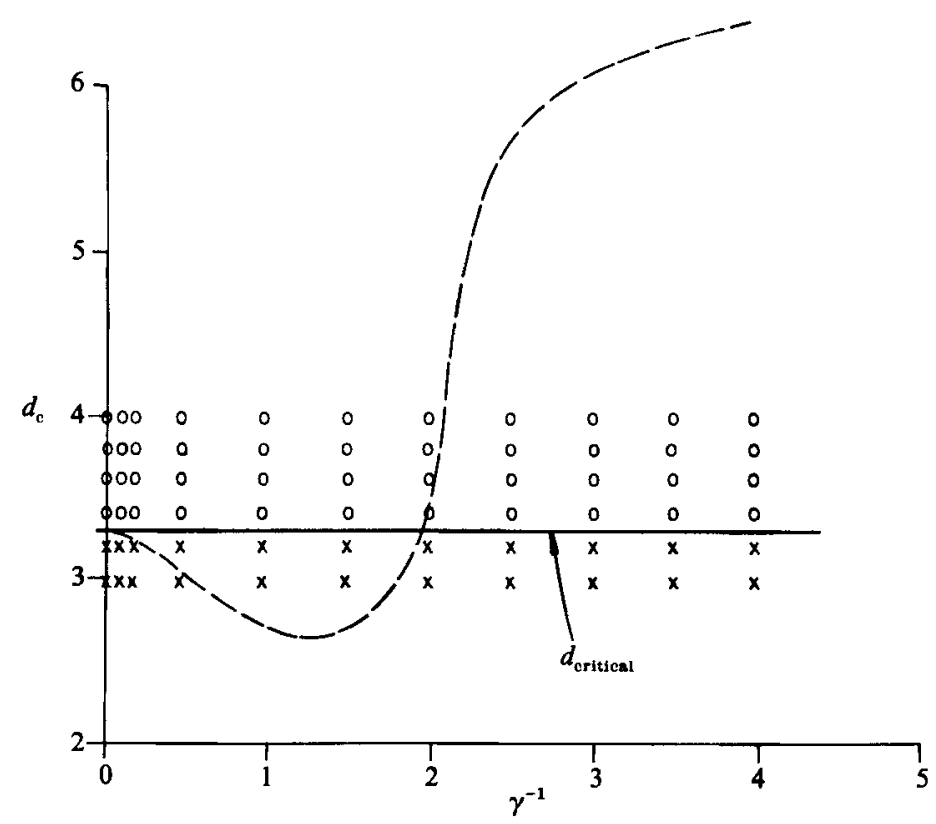

Frgure 12. The critical merger distance as a function of the initial distance $d_{\mathrm{c}}$ and the inverse radius of deformation $\gamma^{-1}$ for $\delta=1$. $\times$ represents a merger and $O$ represents no merger. The dashed line reproduces the experimental results of Griffiths \& Hopfinger (1987).

distribution (1) can be solved exactly in terms of logarithms and modified Bessel functions).

However, on the matter of merger of two vortices in the upper layer, the present computations show that some of the results of Griffiths \& Hopfinger are difficult to reconcile with the predictions of the two-layer quasi-geostrophic model for inviscid initially circular regions of constant geostrophic potential vorticity. In figure 12 we have plotted their curve for the critical merger distance as a function of $\gamma^{-1}$.

We have already pointed out that, as can be seen from (1), in the limit of vanishing $\gamma$ the two layers become decoupled and act as two independent two-dimensional Euler systems. Therefore as $\gamma \rightarrow 0$ the critical merger distance for vortices in the upper layer should be equal to the Euler value - i.e. 3.3. The fact that the results of Griffiths \& Hopfinger are in disagreement with this prediction (at least for the range in $\gamma$ for which the experiments were performed) seems to indicate that at small $\gamma$ their system was not reproducing the dynamics of constant-potential-vorticity nearly-inviscid quasi-geostrophic finite-area vortices.

We have ourselves conducted numerical experiments to determine how the critical merger distances changes as $\gamma$ is varied from zero to infinity. We have proceeded as follows. At $t=0$ the geostrophic potential vorticity distribution is composed of two circles of radius 1 separated by a distance $d_{\mathrm{c}}$. The geostrophic potential vorticity is constant within each circle, and, without loss of generality, we can take its value to be equal to 1 . Each contour is discretized by a finite number of nodes. To follow the evolution of the contours, each node is advected in a Lagrangian fashion according to

$$
\frac{\mathrm{d} X_{k}}{\mathrm{~d} t}=u, \quad \frac{\mathrm{d} Y_{k}}{\mathrm{~d} t}=v
$$




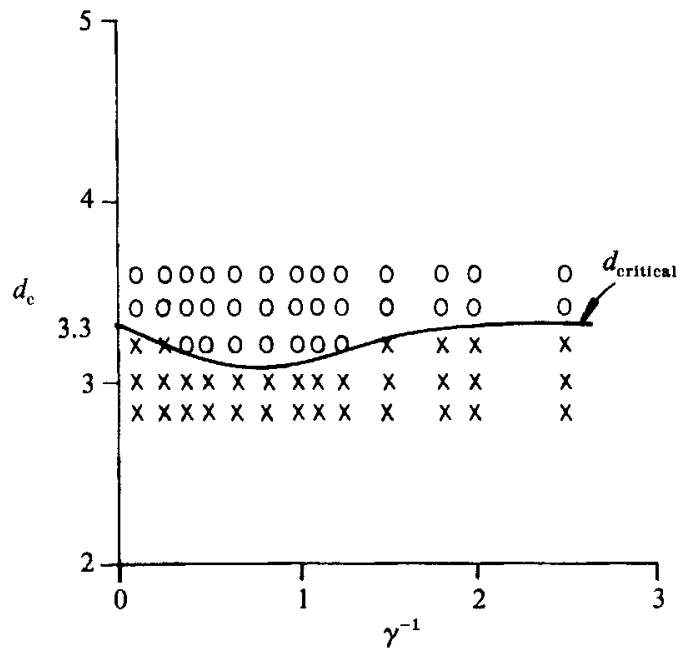

Figure 13. The critical merger distance as a function of the initial distance $d_{\mathrm{c}}$ and the inverse radius of deformation $\gamma^{-1}$ for $\delta=0.2 \times$ represents a merger and $\bigcirc$ represents no merger.

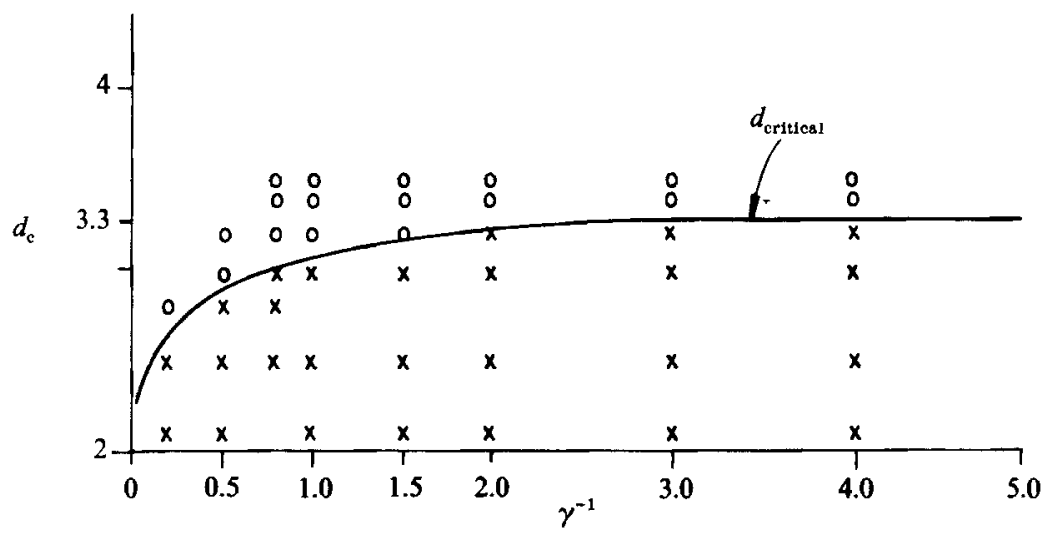

Figure 14. The critical merger distance as a function of the initial distance $d_{\mathrm{c}}$ and the inverse radius of deformation $\gamma^{-1}$ for $\delta=0 . \times$ represents a merger and $O$ represents no merger.

where $\left(X_{k}, Y_{k}\right)$ is the position of node $k$, and $u$ and $v$ are calculated by evaluating the contour integrals in (5). We have used a simple fourth-order Runge-Kutta scheme for the time-stepping. Since we are only interested in determining whether the two vortices merge, every few time-steps we perform a test that stops the run as soon as contours have crossed, a sure sign that merger has occurred. We decide that no merger occurs after the two vortices have revolved around each other for a few times.

Our results are also shown in figure 12. They were obtained by repeating the initialvalue problem for many different values of $d_{\mathrm{c}}$ and $\gamma$. The line for the critical merger distance was drawn in by hand and it serves the purpose of visually separating the region of merger from the region of no merger.

It may at first seem surprising that over the whole range of $\gamma$, the critical merger distance is contained in the interval between 3.2 and 3.4. However, this not only reproduces the expected Euler values for large and small $\gamma$, but is also in qualitative agreement with the results of the previous sections for the $V$-states, in which we have 
shown that, for $\delta=1$, the solutions are remarkably close to the Euler case over the entire $\gamma$-range.

In order to lend support to this interpretation we have conducted similar experiments for two different values of $\delta$. In figure 13 the results for $\delta=0.2$ are shown. As expected from our knowledge of the $\mathrm{V}$-states the deviations from the Euler case are more substantial and take place where $\gamma$ is $O(1)$. The qualitative direction of the critical distance curve is easily understood from our knowledge of the Green function. When $\gamma$ is $O(1)$ the $K_{0}$ contribution to the Green function is felt most strongly, and is reflected in the fact that the two vortices interact less strongly than if the Green function were a pure logarithm; it is thus necessary to put them closer at $t=0$ (i.e. decrease $d_{c}$ ) if merger is to occur.

Finally, for the equivalent barotropic case $\delta=0$, the results are shown in figure 14 . Once again we see that, in that model, the large- $\gamma$ limit exhibits a behaviour qualitatively different from the finite- $d$ cases, because the vortices are exponentially shielded from each other as $\gamma$ becomes larger than $O(1)$.

\section{Non-axisymmetrization and suppression of filamentation in the equivalent barotropic model at large $\gamma$}

For the purpose of understanding whether and to what extent the findings of Melander et al. (1987a) concerning axisymmetrization through filamentation apply to the two-layer system of interest in this study (i.e. one with no geostrophic potential vorticity in the lower layer) we have examined a few cases of layer merger at higher resolution.

In order to follow the evolution far enough in time, it becomes necessary to readjust the nodes representing the discretized contour every few time steps. The criterion we have chosen is based on the idea that more resolution is needed where the curvature is the greatest. More specifically we have decided to place the nodes along the contour in such a way that their density is directly proportional to the local curvature raised to the power $\frac{1}{3}$; this value was chosen because it can be shown to be optimal for the Kirchhoff ellipse (Zou et al. 1988). For more details on the algorithm used, refer to Polvani (1988).

We now present a series of high-resolution mergers for which $d_{c}=2.2$. The number of nodes varies as the contours evolve and usually increases drastically when filaments are formed. In these runs the number of nodes in each contour is usually of the order of a few hundred. Because of the symmetry of the problem, only one contour needs to be computed at each time step, the other one being obtained by reflecting the first about the origin.

In figure 15 the merger for $\delta=0$ and $\gamma=10^{-3}$ is shown. This is a pure Euler merger. Note that long thin vorticity filaments are shed as the two vortices coalesce to form a single vortex. Compare this with the merger at $\delta=0$ but $\gamma=1$, shown in figure 16. The overall qualitative behaviour is not dissimilar, with the exception that for $\gamma=1$, the vorticity in the filaments 'rolls up' into small pools. Thus, in contrast to the Euler case, the final state is really composed of three vortices instead of a vortex surrounded by filaments.

This roll-up behaviour, as distinct from the continual elongation of the filaments present in the Euler case, is probably related to a similar phenomenon observed by Juckes \& McIntyre (1987) in high-resolution one-layer spectral simulations of the polar vortex. A qualitatively similar result has also recently been obtained by Williams \& Wilson (1988) in their finite-difference simulation of Jovian vortices (cf. 
$t=0$

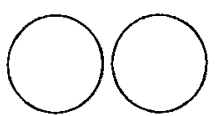

3
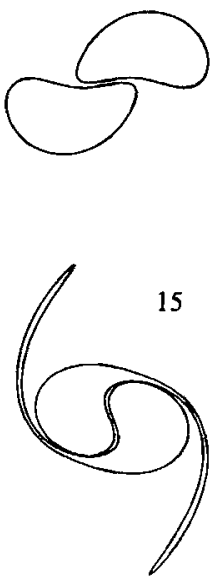

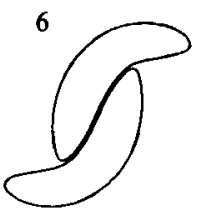

9
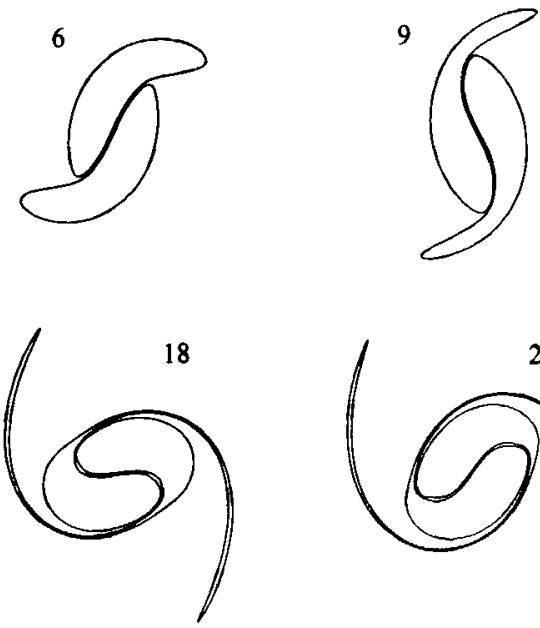

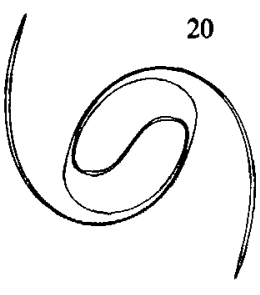

Figure 15. Merger of two initially circular regions of equal potential vorticity for $\delta=0$ and $\gamma=0.001$. The initial distance $d_{\mathrm{c}}=2.2$.

$t=0$

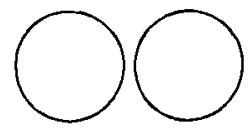

4

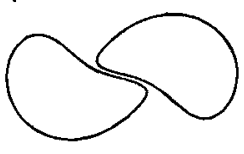

8

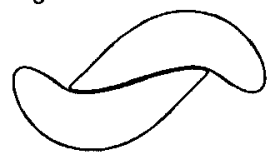

20
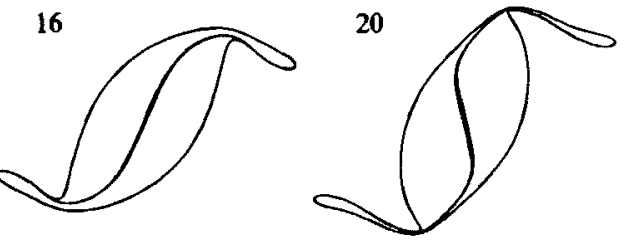

32

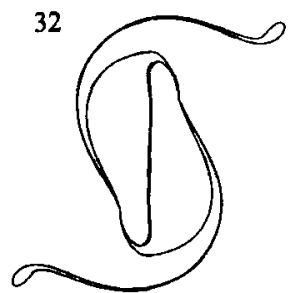

Figure 16. Merger of two initially circular regions of equal potential vorticity for $\delta=0$ and $\gamma=1$. The initial distance $d_{c}=2.2$.

in particular their figure 7). Although their system is not quasi-geostrophic (they solved the full shallow-water equations with one layer), their parameter ranges are such that geostrophy is the prevailing balance in the flow.

Maintaining $\delta=0$ we show the case $\gamma=3$ in figure 17. Observe how drastically different the behaviour is at this higher value of $\gamma$. In this case no filaments are formed and the vorticity does not axisymmetrize. This is undoubtedly a result of the fact that the Green function has no logarithmic component (this is the equivalent 

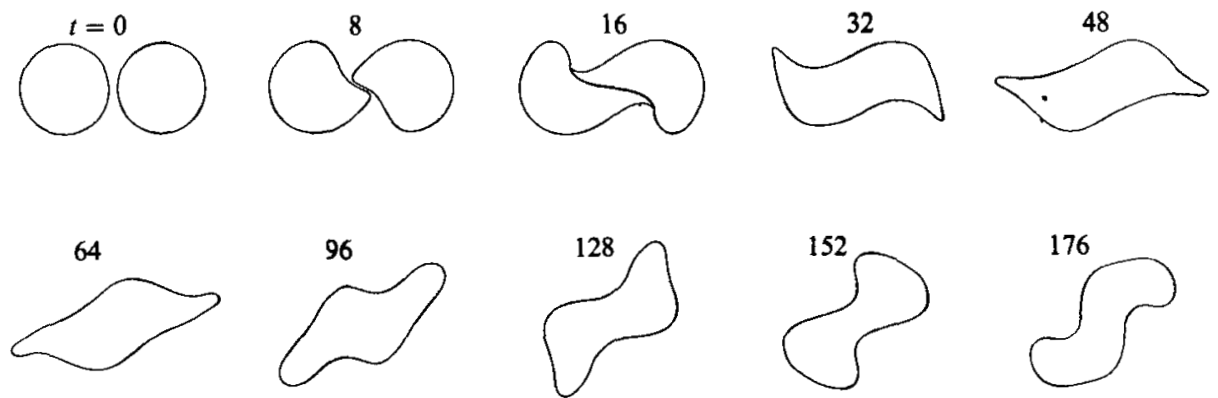

Figure 17. Merger of two initially circular regions of equal potential vorticity for $\delta=0$ and $y=3$. The initial distance $d_{\mathrm{c}}=2.2$.
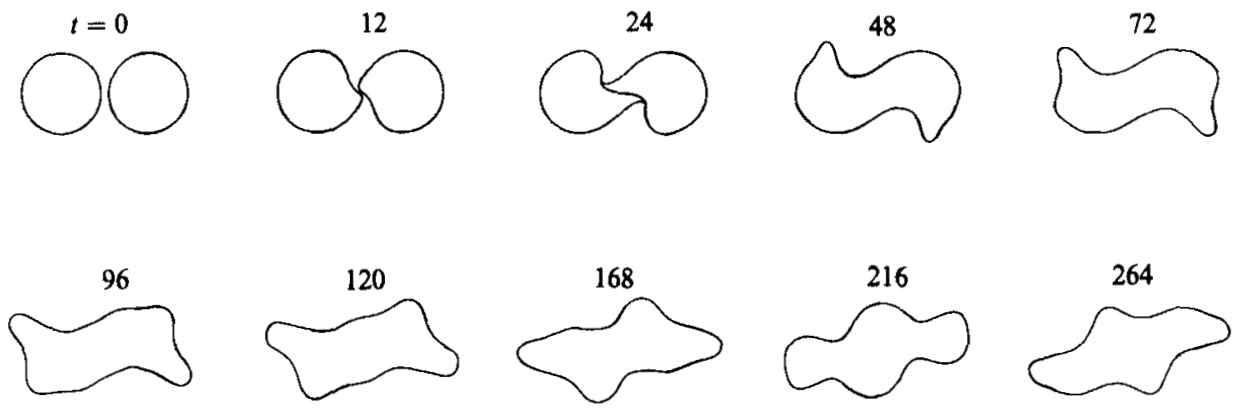

Figure 18. Merger of two initially circular regions of equal potential vorticity for $\delta=0$ and $\gamma=5$. The initial distance $d_{\mathrm{c}}=\mathbf{2 . 2}$.
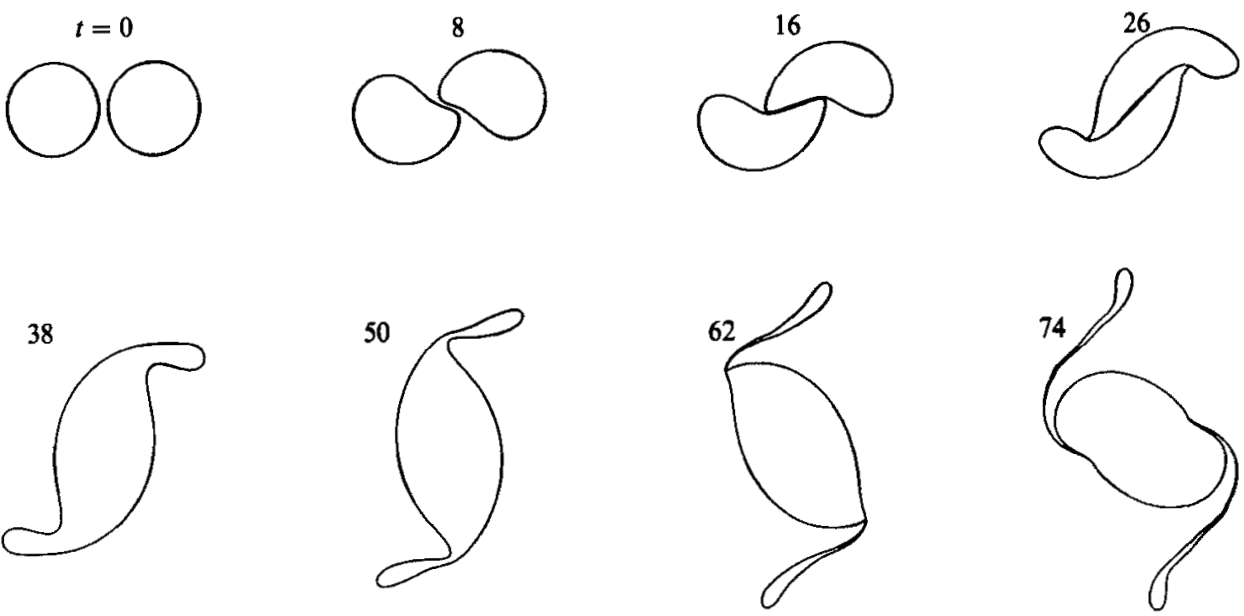

Figure 19. Merger of two initially circular regions of equal potential vorticity for $\delta=0.2$ and $\gamma=5$. The initial distance $d_{\mathrm{c}}=\mathbf{2 . 2}$.

barotropic case $\delta=0$ ). For computational purposes a 'surgery' was practiced on the contours at $t=26$; the common portion of the contours that were sliding along each other was removed, and after the surgery only one contour is left. The surgery has no effect on the qualitative behaviour of the evolution (we have verified this by doing the surgery at slightly different times and comparing the results).

The same non-axisymmetrizing behaviour can be observed in figure 18 for $\gamma=5$ 
(and still $\delta=0$ ). Here again no filaments of vorticity are shed and instead largeamplitude non-breaking nonlinear waves are seen to propagate on the boundary of the vortex. Note that the overall rotation of the vortex is extremely small when compared with the Euler case, and this is a direct consequence of the fact that for a $K_{0}$ Green function at large $\gamma$ most of the vortex interaction is very local. This is most apparent at the beginning of the merger, when only the portions of the vortices that are close enough to feel the influence of the other vortex undergo any deformation. A surgery was performed in this run at $t=24$.

We mention that D. G. Dritschel (private communication) has recently performed much higher resolution contour-surgical computations with the equivalent barotropic model, and confirmed the suppression of filamentation at large scales (i.e. comparable with the radius of deformation). This suppression can also be understood from an analysis of the stagnation points of the corotating stream function (Polvani, Flierl \& Zabusky $1988 b$ ).

Finally, the merger for $d_{c}=2.2, \gamma=5$ but $\delta=0.2$ is shown in figure 19. Notice what a dramatic difference the presence of a finite (even if deep) lower layer makes. When $\delta \neq 0$ the presence of the $K_{0}$ Green function is only felt at small distances and manifests itself in the roll-up of vorticity in the filaments, but the gross features of the evolution are dominated by the logarithmic component of the Green function.

\section{Merger and doubly connected rotating V-states}

We conclude this investigation of the upper-layer dynamies of the two-layer model by showing that the shapes of the critical merger curves as functions of the radius of deformation (cf. figures 12, 13 and 14) are intimately related to the existence and stability of corotating vortex-pair equilibria, which we designate hereafter with the term 'doubly connected upper-layer rotating $V$-states', illustrated schematically in figure 20 .

These $V$-states are composed of two identical vortices in the upper layer, with equal and constant geostrophic potential vorticity, rotating around their common centroid with constant angular velocity and without change in shape. The only parameter needed to describe them is $\nu \equiv x_{A} / x_{B}(0<v<1)$, the ratio of the inner to outer radius of each vortex (see figure 20). For each value of $v$ one can calculate, from the shape of the $V$-state, the distance between the centroids of the two vortices and an equivalent radius for each vortex (defined as the square-root of the area divided by $\pi$ ). The ratio, $d_{v}$, of these two quantities is then a function of $\nu, \gamma$ and $\delta$.

Consider how the shapes of the vortices evolve as $\nu$ is varied from 1 to 0 keeping the properties of the two-layer model unchanged (i.e. at fixed $\gamma$ and $\delta$ ). For $\nu$ near 1 the vortices are nearly circulart, and the ratio of their distance to their radius, $d_{v}$, is very large. As $\nu$ is decreased the vortices must become more elliptical to resist each other's shear and remain in equilibrium, and $d_{\mathrm{v}}$ decreases as $y$ does. The last equilibrium to be found is the one at $\nu=0$ when the two vortices are actually touching at one point; this is commonly referred to as the 'limiting' $\mathrm{V}$-state ( Wu et al. 1984).

The point of interest is that for the limiting $V$-state the value of $d_{\mathrm{v}}$ is finite, and assumes the minimum value of all $\nu$. In two dimensions, Saffman \& Szeto (1980) showed how the limiting value of $d_{\mathrm{v}}$ (approximately 3.16) is, in fact, extremely close to the critical distance for circular merger (approximately 3.3). The interpretation of

$\dagger$ This was proven analytically by Dritschel (1985) for the two-dimensional Euler equations $(\gamma=0)$. 


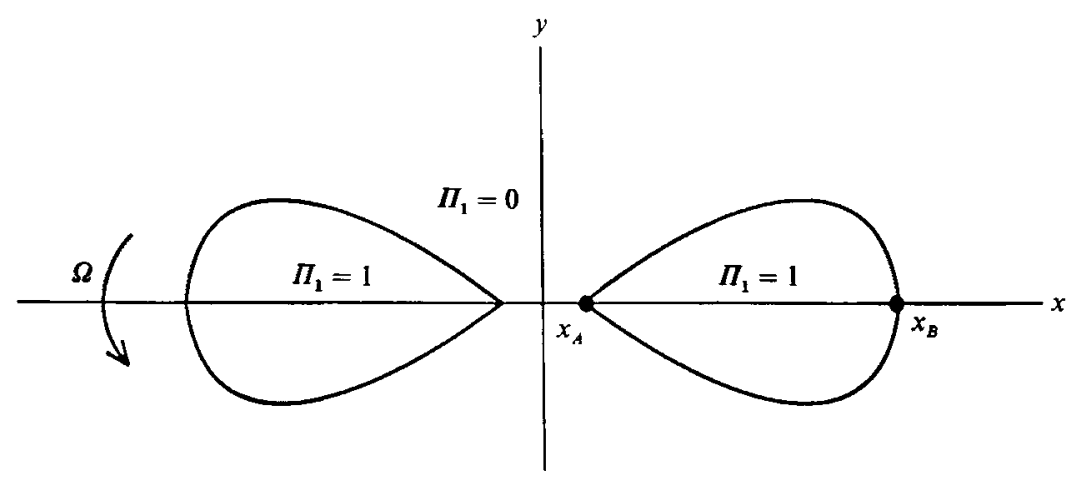

FIGURE 20. Schematic drawing of the geostrophic potential vorticity distribution in the upper layer for a doubly connected rotating $\mathrm{V}$-state.

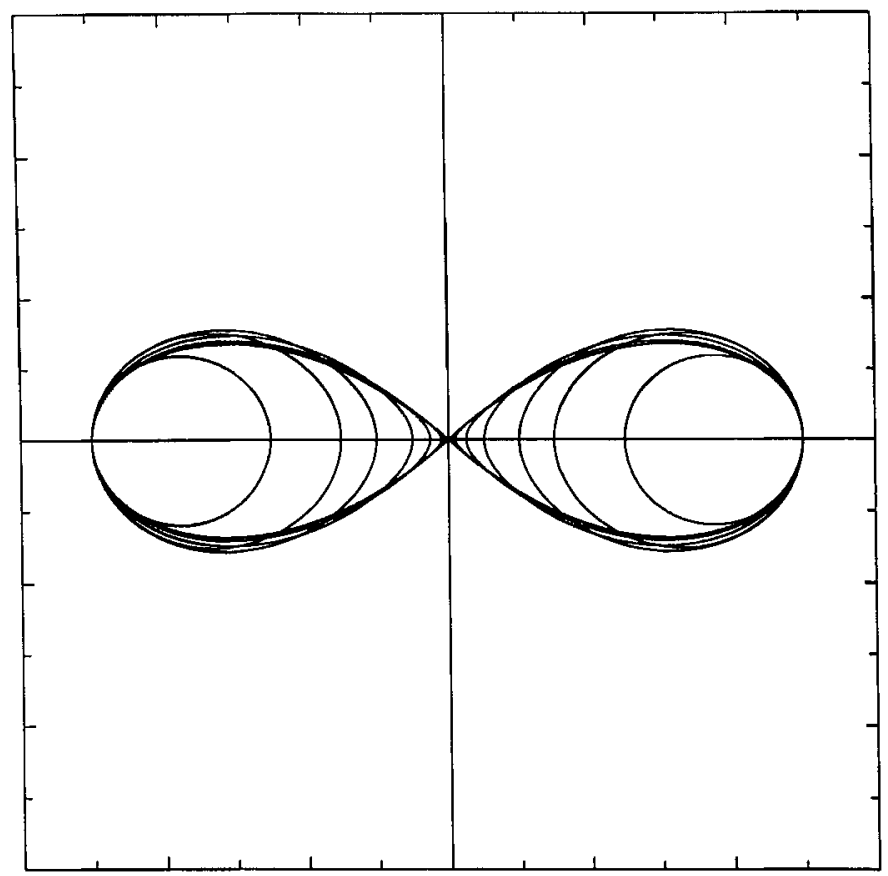

Figcre 21. Doubly connected upper-layer V-states for $\delta=1, \gamma=1$ and several values of $\nu$. As $\nu \rightarrow 0$ the shape becomes more elongated. Some geometrical properties are given in table 1 .

this result is that the initial condition of a merger experiment can be considered to be a perturbation of a doubly connected rotating $\mathrm{V}$-state, if one exists, around that value of $d_{\mathrm{v}}$. If none exists (or one does but is unstable) merger occurs; otherwise, the vortices pulsate and rotate around each other, oscillating around the underlying stable stationary $\mathrm{V}$-state. The fact that, for the two-dimensional Euler equations, the limiting value of $d_{\mathrm{v}}$ is close to the critical merger distance suggests that most doubly connected Euler V-states are actually stable, with the exception of the few 'cuspy' ones near $\nu=0$ (in the linear analysis of Dritschel 1985 instability appears for $\nu<0.083$ ).

We now generalize the idea of Saffman \& Szeto to the two-layer geostrophic model, and show that the results of $\S 5$ are strongly related to the existence of doubly 


\begin{tabular}{cccccc}
\hline$\nu$ & $\Omega$ & $d_{\mathrm{v}}$ & $R$ & $x$ & Area \\
0.500 & 0.01669 & $\mathbf{6 . 1 5 1}$ & 0.244 & 0.751 & 0.187 \\
$\mathbf{0 . 3 0 0}$ & $\mathbf{0 . 0 4 1 7 5}$ & $\mathbf{4 . 0 2 3}$ & 0.325 & 0.653 & 0.331 \\
0.200 & 0.05928 & $\mathbf{3 . 4 5 8}$ & 0.352 & 0.608 & 0.388 \\
0.100 & 0.07572 & 3.160 & 0.360 & 0.569 & 0.408 \\
0.050 & 0.08024 & 3.125 & 0.356 & 0.557 & 0.399 \\
0.020 & $\mathbf{0 . 0 8 0 7 8}$ & 3.140 & 0.353 & 0.554 & 0.391 \\
0.010 & 0.08064 & 3.146 & 0.352 & 0.554 & 0.390 \\
0.005 & 0.08057 & 3.148 & 0.352 & 0.554 & 0.389 \\
0.002 & 0.08053 & 3.149 & 0.352 & 0.554 & 0.389 \\
0.001 & 0.08053 & 3.149 & 0.352 & 0.554 & 0.389
\end{tabular}

TABLE 1. Some properties of doubly connected upper-layer V-states for $\gamma=1, \delta=1$.

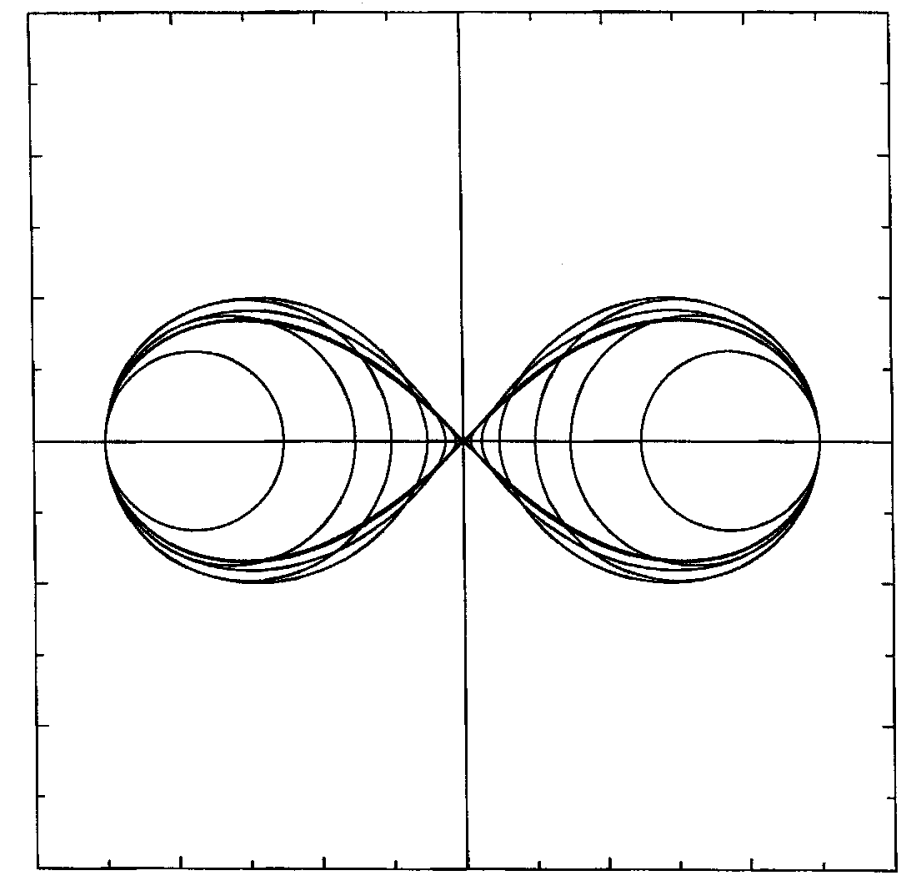

FIGURE 22. As in figure 21 but for $\gamma=10$. Notice that, in general, the shapes are less elongated than at lower $\gamma$. Properties are given in table 2 .

\begin{tabular}{cccccc}
\hline$\nu$ & $\Omega$ & $d_{\mathrm{v}}$ & $R$ & $x$ & Area \\
0.300 & 0.00001 & 3.721 & 0.349 & 0.650 & 0.383 \\
0.200 & $\mathbf{0 . 0 0 0 0 7}$ & $\mathbf{3 . 0 3 4}$ & 0.396 & 0.601 & 0.493 \\
$\mathbf{0 . 1 0 0}$ & $\mathbf{0 . 0 0 0 4 6}$ & $\mathbf{2 . 6 6 6}$ & $\mathbf{0 . 4 2 0}$ & $\mathbf{0 . 5 6 0}$ & $\mathbf{0 . 5 5 5}$ \\
0.050 & $\mathbf{0 . 0 0 0 9 2}$ & $\mathbf{2 . 7 1 3}$ & 0.406 & $\mathbf{0 . 5 5 1}$ & 0.519 \\
0.020 & $\mathbf{0 . 0 0 1 1 7}$ & 2.819 & 0.392 & 0.553 & 0.484 \\
0.010 & $\mathbf{0 . 0 0 1 2 1}$ & 2.848 & 0.389 & 0.555 & 0.477 \\
0.005 & 0.00121 & $\mathbf{2 . 8 5 6}$ & 0.389 & 0.555 & 0.475 \\
0.002 & $\mathbf{0 . 0 0 1 2 0}$ & 2.857 & $\mathbf{0 . 3 8 9}$ & $\mathbf{0 . 5 5 5}$ & $\mathbf{0 . 4 7 5}$ \\
0.001 & 0.00120 & 2.857 & 0.389 & $\mathbf{0 . 5 5 5}$ & 0.475
\end{tabular}

TABLE 2. Some properties of doubly connected upper-layer $V$-states for $\gamma=10, \delta=0$. 

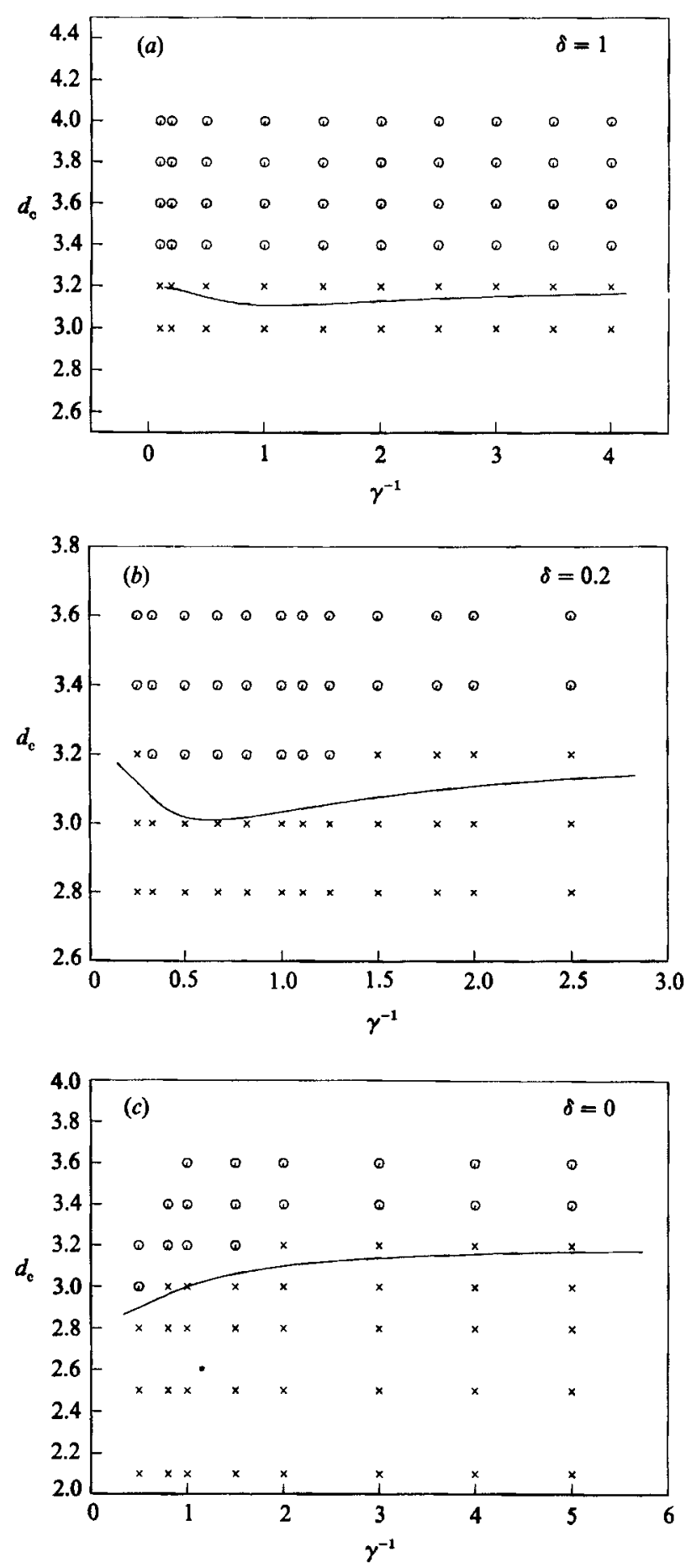

Figure 23. The limiting value of $d_{\mathrm{v}}$ versus $\gamma^{-1}$ for $\delta=1,0.2$ and 0 . The dots and crosses (no merger and merger) are the results of the initial-value problems described in $\$ 5$. 
connected upper-layer rotating $V$-states. These were calculated using appropriate modifications of the same algorithm with which the $V$-states of $\$ 3$ and 4 were obtained. For further details on how we handled the very high curvatures that appear as the limiting $V$-state is approached, refer to Polvani (1988).

As a first example we show in figure 21, a sequence of $V$-states at $\gamma=1$ and $\delta=1$ for several values of $\nu$. A few of their properties are given in table 1, including the angular velocity $\Omega$, the area, the equivalent radius and the centroid location $(\bar{x})$ of each vortex, and the ratio $d_{\mathrm{v}}$ of the intercentroid distance to the equivalent radius. A second example (figure 22) shows the family of doubly-connected equivalent barotropic $(\delta=0)$ rotating $V$-states at $\gamma=10$ (see table 2 for properties of these V-states).

Since our main objective is to relate the existence of these $\mathrm{V}$-states to the merger problem, we have concentrated our attention on the limiting V-states. In figures $23(a)$, $23(b)$ and $23(c)$ we present the curves for the limiting value of $d_{\mathrm{v}}$ versus $\gamma^{-1}$ for the three values $\delta=1.0,0.2$ and 0 , respectively $\dagger$ (we have plotted on those figures the results of the initial-value experiments given in $\$ 5$ ).

Notice how good the qualitative and quantitative agreement is, although, unfortunately, to reduce the computational task, we have had to use a rather coarse grid for the initial-value problems. For the case of equal layers $(\delta=1)$ the limiting value of $d_{\mathrm{v}}$ lies between 3.11 and 3.19 over the entire $\gamma$-range, and is entirely contained within a single grid spacing in $d_{\mathrm{c}}$. For $\delta=0.2$ the limiting value of $d_{\mathrm{v}}$ varies between 3.0 and 3.2, which agrees well with the range of variation of the critical merger distance. Moreover the location of the minimum in $\gamma$ (see figure $23 b$ ), which occurs near $\gamma=0.6$, is in good agreement with the coarse result of the initial-value computations.

In general, as for the Euler $(\gamma=0)$ case, the limiting value of $d_{v}$ is smaller than the critical merger distance and this is easily understood as the consequence of the probable instability of the cuspy near-limiting $\mathrm{V}$-states.

In conclusion we would like to restate in a different, and perhaps more illuminating way, what we believe to be the fundamental reason for the existence of a critical distance in the merger problem : when two vortices are sufficiently separated they fail to merge because of the presence 'in the vicinity' (in the phase space of the system with a proper measure) of a stationary and stable configuration of vorticity that acts as an attractor for the vorticity field, preventing the collapse of the two vortices into a single one. The collapse inevitably occurs when the initial condition is 'far' from any stable doubly connected stationary state; in that case a single vortex constitutes the 'nearest' equilibrium and merger occurs. We shall show, in the second paper, how this idea generalizes to the problem of alignment of two vortices in different layers.

\section{Conclusion}

Three main results have emerged from the study of the $V$-states and merger in the upper layer of a two-layer quasi-geostrophic system. The first is that the upper-layer dynamics in the presence of a lower layer containing no geostrophic potential vorticity is remarkably similar to two-dimensional inviscid Euler dynamies. Second, we have shown that the critical distances for merger of two vortices in the upper layer are very closely related to the existence and geometrical properties of

$\dagger$ Before plotting, the values of $d_{\mathrm{v}}$ and $\gamma$ need to be rescaled to obtain the corresponding values for vortices of area $\pi$ (recall that the merger experiments were conducted with circular vortices of radius 1 ). 
equilibrium corotating vortex pairs in the parameter space spanned by the radius of deformation $(\gamma)$, the ratio of the two layers at rest $(\delta)$ and the initial intercentroid distance $\left(d_{\mathrm{c}}\right)$.

The third conclusion concerns the singular nature of the equivalent barotropic model. We have shown that the dynamics of motions on scales of a few times the radius of deformation are qualitatively very different from those of a two-layer system with a finite, even if very deep, lower layer with constant potential vorticity. In particular, the process of filamentation was found to be greatly suppressed (at least on scales comparable with the radius of deformation), and thus axisymmetrization almost totally inhibited.

In this study the geostrophic potential vorticity was confined to the upper layer alone. In the next paper we shall consider the dynamics of the same two-layer system with geostrophic potential vorticity present in both layers. In particular we shall present the phenomenology of the alignment problem, and show that, analogously to what was presented here for the merger problem, it can be related to the existence of two-layer corotating vortex equilibria.

This work was started at the Woods Hole Geophysical Fluid Dynamies Summer Program during the Summer of 1986. The authors wish to thank Mr A. Maffei for his generous donation of computing facilities during the initial phases of this work, and acknowledge the contribution of Dr E. A. Overman for portions of the contourdynamics algorithm used in this study. The high-resolution computations were performed on the Cray-1 computer of the National Center for Atmospheric Research, which is supported by the National Science Foundation.

\section{Appendix}

Consider a front that separates the plane into two regions of constant potential vorticity as shown in figure 24 . The front is located at $y=L(x, t)$. In the equivalent barotropic formulation the dimensional stream function $\psi(x, y, t)$ must satisfy

$$
\nabla^{2} \psi_{ \pm}-\frac{1}{L_{R}^{2}} \psi_{ \pm}= \pm Q_{0} \text { for } y \gtrless L
$$

where $L_{R}$ is the radius of deformation. The boundary conditions at $y=L$ are as follows:

$$
\psi_{+}=\psi_{-}, \quad \boldsymbol{\nabla} \psi_{+} \cdot \hat{\boldsymbol{n}}=\boldsymbol{\nabla} \psi_{-} \cdot \hat{\boldsymbol{n}}
$$

where $\hat{\boldsymbol{n}}$ is the unit normal at the front, and the kinematic condition:

$$
v(x, L, t)=\partial_{t} L+u(x, L, t) \partial_{x} L,
$$

where the velocities $u$ and $v$ are defined in terms of the stream function by

$$
u=-\partial_{y} \psi, \quad v=\partial_{x} \psi
$$

First change variables from $(x, y, t)$ to $(x, \eta, t)$, where $\eta$ is defined by

$$
\eta=y-L(x, t)
$$

In the new coordinate system the vorticity equation (A 1) becomes

$$
\left\{\partial_{x}^{2}+\left(1+L_{x}^{2}\right) \partial_{\eta}^{2}-2 L_{x} \partial_{x} \partial_{\eta}-L_{x x} \partial_{\eta}-\frac{1}{L_{R}^{2}}\right\} \psi_{ \pm}= \pm Q_{0} \text { for } \eta \gtrless 0,
$$

and the kinematic boundary condition simplifies to

$$
L_{t}=\psi_{x} \text { at } \eta=0 .
$$




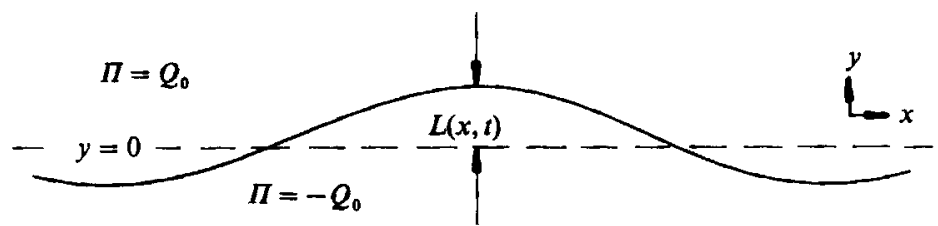

Froune 24. Sketch of a potential vorticity front in the equivalent barotropic model.

Equations (A 4) and (A 5) are dimensional. Next non-dimensionalize as follows:

$$
L, x=O(\mathscr{L}), \quad \eta=O\left(L_{R}\right), \quad \psi=O\left(Q_{0} L_{R}^{2}\right), \quad t=O\left(\frac{1}{\epsilon^{3} Q_{0}}\right)
$$

and obtain the non-dimensional vorticity equation:

$$
\left[\left(1+L_{x}^{2}\right) \partial_{\eta}^{2}-1\right] \psi_{ \pm}-\epsilon\left[2 L_{x} \partial_{x} \partial_{\eta}+L_{x x} \partial_{\eta}\right] \psi_{ \pm}+\epsilon^{2}\left[\partial_{x}^{2}\right] \psi_{ \pm}= \pm 1 \text { for } \eta \gtrless 0,
$$

and the non-dimensional kinematic boundary condition

$$
\epsilon \partial_{t} L=\partial_{x} \psi
$$

In (A 6) and (A 7) all the variables are non-dimensional and the only parameter that appears is

$$
\epsilon=\frac{L_{R}}{\mathscr{L}}=\frac{1}{\gamma}
$$

Consider now the case $\epsilon \ll 1$ (i.e. the large- $\gamma$ limit). Write $\psi$ as an asymptotic series:

$$
\psi_{ \pm}=\Psi_{ \pm}+\epsilon \phi_{ \pm}+O\left(\epsilon^{2}\right)
$$

Substituting (A 8) into (A 6) and (A 7) and collecting like powers of $\varepsilon$ one obtains a sequence of problems. At lowest order one has

$$
\left[\left(1+L_{x}^{2}\right) \partial_{\eta}^{2}-1\right] \Psi_{ \pm}= \pm 1 \quad \text { for } \quad \eta \gtrless 0
$$

together with the boundary conditions

$$
\Psi_{+}=\Psi_{-}, \quad \partial_{\eta} \Psi_{+}=\partial_{\eta} \Psi_{-} \quad \text { at } \eta=0 .
$$

System (A 9) and (A 10) is easily solved to yield

$$
\Psi_{ \pm}=\mp\left(1-\mathrm{e}^{\mp s \eta}\right) \text { for } \eta \gtrless 0
$$

where $s$ is defined by

$$
s^{-2}=1+L_{x}^{2}
$$

At next order the following problem emerges:

$$
\left[\left(1+L_{x}^{2}\right) \partial_{\eta}^{2}-1\right] \phi_{ \pm}=2 L_{x} \Psi_{ \pm x \eta}+L_{x x} \Psi_{ \pm \eta} \text { for } \eta \gtrless 0,
$$

together with conditions

$$
\phi_{+}=\phi_{-}, \quad \partial_{\eta} \phi_{+}=\partial_{\eta} \phi_{-} \quad \text { at } \quad \eta=0,
$$

and

$$
\phi_{x}=L_{t} \quad \text { at } \quad \eta=0 \text {. }
$$

The solution of (A 11) and (A 12) is straightforward, and, after substituting it into (A 13) yields the equivalent barotropic long-wave equation

$$
L_{t}=\frac{1}{2}\left(\frac{L_{x}}{\left[1+L_{x}^{2}\right]^{\frac{1}{2}}}\right) \text {. }
$$




\section{REFERENCES}

Deem, G. S. \& ZABusky, N. J. 1978a Stationary V-states : interactions recurrence and breaking. Phys. Rev. Lett. 40, 859.

Deem, G. S. \& ZABUSKY, N. J. $1978 b$ Stationary V-states: interactions recurrence and breaking. In Solitons in Action (ed. K. Longren \& A. Scott), pp. 277-293. Academic.

Dritschel, D. G. 1985 The stability and energetics of corotating uniform vortices. J. Fluid Mech. 157, 95-134.

DRITSCHEL, D. G. 1988 Contour surgery: a contour dynamies method for long-time behaviour of two-dimensional, inviscid, rotational flow. J. Comput. Phys. 77, 240-266.

GRIFFITHS, R. W. \& HopfINGeR, E. J. 1986 Experiments with baroclinic vortex pairs in a rotating fluid. J. Fluid Mech. 173, 501-518.

GRIFfITHs, R. W. \& Hopfinger, E. J. 1987 Coalescing geostrophic vortices. J. Fluid Mech. 178, $73-79$.

GRYANIK, V. M. 1983 Dynamics of singular geostrophic vortices in a two-level model of the atmosphere (or the ocean). Izv. Akad. Nauk. SSSR Atmos. Ocean. Phys. 19, 171-179.

Hoga, N. \& Stommel, H. 1985 The heton, an elementary interaction between discrete baroclinic geostrophic vortices and its implications concerning eddy heat-flow. Proc. R. Soc. Lond. A 397, $1-20$.

Juckes, M. N. \& McIntyre, M. E. 1987 A high resolution one layer model of breaking planetary waves in the stratosphere. Nature 328, 590-596.

McWiLLIAMs, J. C. 1984 The emergence of isolated coherent vortices in turbulent flow. J. Fluid Mech. 146, 21-43.

MoWilliams, J. C. 1989 Statistical properties of decaying geostrophic turbulence. J. Fluid Mech. 198, $199-230$.

Melander, M. V., MoWilliams, J. C. \& Zabusky, N. J. 1987 a Axisymmetrization and vorticity gradient intensification of an isolated two-dimensional vortex through filamentation. J. Fluid Mech. 178, 137-159.

Melander, M. V., Zabusky, N. J. \& McWilliams, J. C. $1987 b$ Asymmetric vortex merger in two-dimensions: Which vortex is 'victorious'? Phys. Fluids 30, 2610-2612.

Melander, M. V., Zabusky, N. J. \& McWilliams, J. C. 1988 Symmetric vortex merger in twodimensions : causes and conditions. J. Fluid Mech. 195, 303-340.

Melander, M. V., ZABusky, N. J. \& StycZek, A.S. 1986 A moment model for vortex interactions of the two-dimensional Euler equations. Part I. Computational validation of a Hamiltonian elliptical representation. J. Fluid Mech. 167, 95-115.

NorbURy, J. 1973 A family of steady vortex rings. J. Fluid Mech. 57, 417-431.

Overman, E. A. \& Zabusky, N. J. $1982 a$ Evolution and merger of isolated vortex structures. Phys. Fluids 25, 1297-1305.

Overman, E. A. \& ZABUSKY, N. J. $1982 b$ Coaxial scattering of Euler equation translating V-states via contour dynamies. J. Fluid Mech. 125, 187-202.

Peduosky, J. 1979 Geophysical Fluid Dynamics, chap. 6. Springer.

PHILliPs, N. A. 1954 Energy transformations and meridional circulations associated with simple baroclinic waves in a two-level, quasigeostrophic model. Tellus 6, 273-286.

Pierrehumbert, R. T. 1980 A family of steadily translating vortex pairs with distributed vorticity. J. Fluid Mech. 99, 129-144.

Polvani, L. M. 1988 Geostrophic vortex dynamics. Ph.D. thesis, Massachusetts Institute of Technology, 1988.

Polvani, L. M., Flierl, G. R. \& Zabusky, N. J. 1989 Filamentation of coherent vortex structures via separatrix crossing: a quantitative estimate of onset time. Phys. Fluids. A 1 , $181-184$.

Polvani, L. M., Zabusky, N. J. \& Flierl, G. R. 1988 Applications of contour dynamics to twolayer quasi-geostrophic. Fluid Dyn. Res. 3, 422-424.

Pratt, L. J. \& Stern, M. E. 1986 Dynamics of potential vorticity fronts and eddy detachment. J. Phys. Oceanogr. 16, 1101-1120.

Saffman, P. G. \& Schatzman, J. C. 1982 Stabilities of a vortex street of finite vortices. J. Fluid Mech. 117, 171-185. 
Saffman, P. G. \& Szeto, R. 1980 Equilibrium shapes of a pair of equal uniform vortices. Phys. Fluids 23, 2339-2342.

SALMON, R. 1982 Geostrophic turbulence. In Turbolenza e Predicibilità nella Fluidodinamica Geofisica e la Dinamica del Clima. Scuola Internazionale di Fisica Enrico Fermi, LXXXVIII, pp. 113-158.

Williams, G. P. \& Wirson, R. J. 1988 The stability and genesis of Rossby vortices. J. Atmos. Sci. 45, 207-241.

Wu, H. M., Overman, E. A. \& Zabusk Y, N. J. 1984 Steady state solutions of the Euler equation in two dimensions. Rotating and translating $\mathrm{V}$-states with limiting cases. I. Numerical results. J. Comput. Phys. 53, 42-71.

Young, W. R. 1985 Some interactions between small numbers of baroclinic geostrophic vortices. Geophys. Astrophys. Fluid Dyn. 33, 42-71.

Zabusky, N. J., Hughes, M.H. \& Roberts, K. V. 1979 Contour dynamics for the Euler equations in two dimensions. J. Comput. Phys. 30, 96-106.

Zou, Q., Overman, E. A., Wu, H. M. \& Zabusky, N. J. 1988 Contour dynamics for the Euler equations: curvature controlled initial node placement and accuracy. J. Comput. Phys. $\mathbf{7 8}$, $350-372$. 